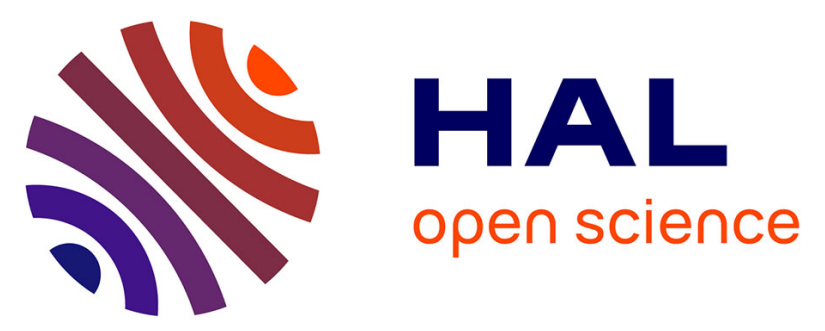

\title{
Estimation of position and resistance of a sensorless PMSM : a nonlinear Luenberger approach for a non-observable system
}

\author{
Pauline Bernard, Laurent Praly
}

\section{- To cite this version:}

Pauline Bernard, Laurent Praly. Estimation of position and resistance of a sensorless PMSM : a nonlinear Luenberger approach for a non-observable system. IEEE Transactions on Automatic Control, 2020, 10.1109/TAC.2020.2981341 . hal-01824171v4

\section{HAL Id: hal-01824171 \\ https://hal.science/hal-01824171v4}

Submitted on 2 Mar 2020

HAL is a multi-disciplinary open access archive for the deposit and dissemination of scientific research documents, whether they are published or not. The documents may come from teaching and research institutions in France or abroad, or from public or private research centers.
L'archive ouverte pluridisciplinaire HAL, est destinée au dépôt et à la diffusion de documents scientifiques de niveau recherche, publiés ou non, émanant des établissements d'enseignement et de recherche français ou étrangers, des laboratoires publics ou privés. 


\title{
Estimation of position and resistance of a sensorless PMSM : a nonlinear Luenberger approach for a non-observable system
}

\author{
Pauline Bernard and Laurent Praly*
}

March 2, 2020

\begin{abstract}
We show that the pair (rotor position, resistance) of a non-salient Permanent Magnet Synchronous Motor (PMSM) is not observable in the sensorless setting, namely when only the electrical signals (voltages and intensities) are measured. However, if the rotation speed $\omega$ and the current in rotating frame $i_{d}$ are not constantly zero, we prove that there only exist a maximum of six indistinguishable solutions, the resistance being the root of a polynomial of degree six. Besides, in the particular case where $\omega, i_{d}$ and $i_{q}$ are constant, we show that the number of possible solutions is reduced to two, with two well-identified possible values for the resistance. Those two solutions can be dissociated if the sign of $i_{q}$ (namely the mode of use of the motor) is known. Inspired by the nonlinear Luenberger methodology, this property enables us to propose an estimation algorithm which relies on a one-dimensional minimization of a criterion involving the voltages and intensities filtered at three distinct sufficiently large frequencies. Its performances are tested and illustrated in simulations on real data.
\end{abstract}

\section{Introduction}

For an observer to give an asymptotically converging estimate of the system state, this system must be detectable if not observable. So for any application, before attempting any design of an observer, observability should be studied. The application we are concerned with in this paper is the estimation of the rotor position of a non-salient Permanent Magnet Synchronous Motors (PMSM) with known inductance $L$, known magnet flux $\Phi$, but unknown stator resistance $R$, and in the so-called sensorless setting, namely when only electric variables - currents and voltages - are measured.

${ }^{*}$ P. Bernard and L. Praly are with MINES ParisTech, PSL Research University, Paris, France. emails:pauline.bernard@mines-paristech.fr, laurent.praly@mines-paristech.fr. 


\subsection{Context}

In order to reduce their cost and increase their sobriety and reliability, Permanent Magnet Synchronous Motors (PMSM) are not always equipped with mechanical sensors. Their control and supervision thus require observers to estimate the mechanical variables - rotor position and speedfrom the measurement of the electric variables only, and using a model of the dynamics which typically involves $R, L$ and $\Phi$.

A review of the first steps towards sensorless control was given in [1] and a Luenberger observer was proposed in [2] in order to estimate the position of a PMSM from electrical measurements only. Then, in [3], pursuing the same goal, was proposed a very simple gradient observer which turned out to be extremely effective in practice. However, from a theoretical view point, it was proved in [4] to be only conditionally convergent: it may admit several equilibrium points depending on the rotation speed $\omega$. Fortunately, later in [5], the authors showed how a minor modification enables to achieve global asymptotic stability thanks to convexity arguments.

All these observers typically require the knowledge of the parameters $R, \Phi$ and $L$. Unfortunately, while $L$ may be considered known and constant (as long as there is no magnetic saturation), $R$ and $\Phi$ do vary significantly with the temperature and these variations should be taken into account in the observer. That is why efforts have been made to look for position observers which do not rely on the knowledge of those parameters, or even better, which also estimates them. For instance, $[6,7]$ have proposed sensorless position observers which are independent from the magnet flux. This line of research was completed in [8] by directly extending the gradient observer proposed in [3] with the estimation of $\Phi$. But as far as the resistance is concerned, we are not aware of any sensorless observation algorithm with proved global convergence. A first attempt appeared in [9] using an adaptive sliding mode scheme. Then, in [10, 11], and recently in [12, 13] observers/control for PMSMs with unknown resistance were proposed, but guaranteeing only local stability. In [14], the authors proposed a global adaptive observer to make the gradient observer from [3] independent from the resistance, but only a qualitative convergence to a neighborhood of the solution is ensured; quantitatively, there is no control on the size of this neighborhood.

Actually, along those attempts at observer design, we have not found any report on the study of observability of the pair (rotor position, resistance) for a PMSM. Some observability studies were carried out in the context of induction motors for instance in $[15,16,17]$, the latter two exhibiting pairs of indistinguishable trajectories in steady state when the torque or stator resistance is unknown, thus explaining why most control/observer designs with parameter adaptations are inherently local. We show in this paper that, in accordance with those observations for induction machines, a PMSM with unknown resistance is not observable unless other information is added (see also [18]). Nevertheless, it is a peculiar kind of non observability because we prove that when the rotation speed and the current in rotating frame $i_{d}$ are not constantly zero, there are at most

six possible values for the pair (rotor position, resistance). Besides, when the rotation speed and 
the currents evaluated in the rotating frame are constant, the number of indistinguishable solutions is reduced to two, and can be dissociated thanks to the extra knowledge of the motor mode of use (motor or generator). This finiteness of the classes of indistinguishability, already described in $[16,17]$ could not appear in a linear context where non observability necessarily implies an infinite number of indistinguishable trajectories.

But then, can an observer be proposed when the system has classes of indistinguishability with finite cardinality? A positive answer was given in [16, 19] when the system can be written in an "observable-like" form which, due to unobservability, involves a set valued map that is considered in the design as an unknown input. Here we propose another positive answer of a completely different nature, relying on a nonlinear Luenberger design. This design consists in transforming the system dynamics into asymptotically stable linear dynamics with the measurements as input, and this, independently from the given system observability. Then, an estimate is obtained by inverting the transformation, invertibility being here related to observability. This approach was first developed by Luenberger himself for linear systems in [20], then extended to stationary nonlinear systems ([21, 22 ] and references therein), and finally to time-varying systems in [23, 24]. It was also successfully applied to the sensorless position estimation of a PMSM (with known resistance) in [5]. In all those papers, this design is used for observable systems where the invertibility of the transformation is guaranteed. But we show in this paper that it is still useful in the absence of observability and invertibility. In our particular example, it enables to transform the problem of estimating the pair (rotor position, resistance) from a single output equation valid at all times into the one of solving at each time a set of three equations which are valid only asymptotically as time goes to infinity, but exponentially fast. They involve the voltages and currents filtered at three distinct sufficiently large frequencies. Because of non observability these three equations must have non unique solutions. A key result that we establish is that there are at most six possible values of the pair (rotor position, resistance). So we recover exactly the property of unobservability. Actually these possible solutions can be a posteriori discriminated according to extra knowledge of those quantities, such as bounds for the resistance, mode of use of the motor, etc.

Hence, by going with a Luenberger design, we get an observer with two components

- a dynamic component made of a cascade of linear filters,

- a static component, its output map, delivering the estimate computed from the filters' states. It is a set valued map since its image has in general 6 values.

This is to be compared for instance with the observer in [17] and [18], the dynamics of which are a differential inclusion. 


\subsection{Problem statement}

We consider a balanced three-phase wye-connected PMSM. Using Joule's and Faraday's laws, its equivalent two-phase model expressed in a fixed stator frame reads (see [25, (7.69)])

$$
\dot{\Psi}=u-R i
$$

where $\Psi$ is the total flux generated by the stator windings and the permanent magnet, $(u, i)$ are the voltage and current, in the fixed stator frame, and $R$ the stator winding resistance. The quantities $u, i$ and $\Psi$ are two dimensional vectors. The way the total flux $\Psi$ is related to the rotor angle $\theta_{r}$ differs depending on the geometry of the rotor and stator. When the repartition of the windings and the profile of the magnet are perfectly symmetric, the motor is said to be non-salient and the total flux may be expressed simply as (see [25, (7.72)])

$$
\Psi=L i+\Phi\left(\begin{array}{c}
\cos \theta \\
\sin \theta
\end{array}\right)
$$

where $L$ is the stator inductance, $\Phi$ the magnet's flux, and $\theta=n_{p} \theta_{r}$ the electrical phase, with $n_{p}$ the number of poles (winding pairs) of the stator. Plugging (2) into (1) leads to the usual PMSM model

$$
\begin{aligned}
L \dot{\hat{i}} & =u-R i-\Phi n_{p} \omega_{r}\left(\begin{array}{c}
-\sin \left(n_{p} \theta_{r}\right) \\
\cos \left(n_{p} \theta_{r}\right)
\end{array}\right) \\
\dot{\theta}_{r} & =\omega_{r} \\
\mathcal{I} \dot{\omega}_{r} & =\Phi n_{p} i^{\top}\left(\begin{array}{c}
-\sin \left(n_{p} \theta_{r}\right) \\
\cos \left(n_{p} \theta_{r}\right)
\end{array}\right)-\tau_{L}
\end{aligned}
$$

where $\mathcal{I}$ is the inertia of the rotor and $\tau_{L}$ the load torque. But this operation makes $\omega_{r}=\dot{\theta}_{r}$ appear in the model along with two new parameters $\mathcal{I}$ and $\tau_{L}$ which must be either known or estimated. Fortunately, as noticed in [2], (2) implies

$$
\begin{gathered}
|\Psi-L i|^{2}-\Phi^{2}=0 \\
\theta=\arg (\Psi-L i) .
\end{gathered}
$$

So, according to (5), in the case where $L$ and $i$ are known, an estimate of $\theta$ can be simply recovered from an estimate of the total flux $\Psi$. Therefore, as suggested in [2], it is preferable to consider the model made of (1)-(4) instead of (3a), namely

$$
\dot{\Psi}=u-R i \quad, \quad \Phi^{2}-L^{2}|i|^{2}=\Psi^{\top} \Psi-2 L i^{\top} \Psi
$$

with known inputs $(u, i)$ and where the information given by (4) is used as a measurement. These equations are independent from the parameters $\mathcal{I}$ and $\tau_{L}$. 
It is proved in [8] that it is possible to estimate both $\Psi$ and $\Phi$ at the same time. In this paper, we rather suppose that the flux $\Phi$ produced by the magnet is known, and the resistance $R$ unknown. We want to know if it is possible to estimate both $\Psi$ (and thus $\theta$ ) and $R$.

In Section 2, we show that the knowledge of $(u, i), \Phi$ and $L$ is generally not enough to determine uniquely $\Psi$ and $R$. However, when the rotation speed and the currents evaluated in the rotating frame are non zero, there are at most six possible solutions, which may be distinguished from one another by the extra knowledge of the mode of use of the machine (motor or generator). Then, we exploit this knowledge to propose an estimation algorithm for $(\Psi, R)$ (and thus $(\theta, R)$ ) based on a nonlinear Luenberger observer design in Section 3. In Section 4, we propose a possible practical implementation and study its robustness to measurement and model errors (such as the presence of saliency) in Section 5. We finally illustrate its performance on a simulated scenario, as well as on real data in Section 6 .

\section{Notations}

1. the rotation matrix of angle $\theta$

$$
\mathcal{R}(\theta)=\left(\begin{array}{cc}
\cos \theta & -\sin \theta \\
\sin \theta & \cos \theta
\end{array}\right)
$$

The particular one for $\theta=-\frac{\pi}{2}$ is

$$
J=\mathcal{R}\left(-\frac{\pi}{2}\right)=\left(\begin{array}{cc}
0 & 1 \\
-1 & 0
\end{array}\right)
$$

2. the current in a rotated frame of angle $\theta$

$$
I_{d q}(\theta)=\left(\begin{array}{c}
I_{d}(\theta) \\
I_{q}(\theta)
\end{array}\right)=\mathcal{R}(-\theta) i
$$

Its evaluation along a solution is

$$
i_{d q}(t)=I_{d q}(\theta(t))=\left(\begin{array}{c}
i_{d}(t) \\
i_{q}(t)
\end{array}\right)=\left(\begin{array}{c}
I_{d}(\theta(t)) \\
I_{q}(\theta(t))
\end{array}\right)
$$

3. the angle corresponding to a flux $\Psi$

$$
\Theta(\Psi)=\arg (\Psi-L i)
$$

\section{Observability analysis}

Inspired by (6) we consider the time-varying system

$$
\left\{\begin{aligned}
\dot{x} & =u-x_{3} i \\
\dot{x}_{3} & =0 \\
y=\Phi^{2}-L^{2}|i|^{2} & =x^{\top} x-2 L i^{\top} x
\end{aligned}\right.
$$


with $L, \Phi, u$ and $i$ given. The input time signals $u$ and $i$ are assumed to be consistent with this model, i.e. such that $x=\Psi$ and $x_{3}=R$ is a particular solution. In the following $\left(x(t), x_{3}(t)\right)$ denotes a generic solution whereas $(\Psi(t), R)$ is the notation for this particular solution. In some cases, it will be appropriate to rewrite the last equation of (10) equivalently as

$$
0=|x-L i|^{2}-\Phi^{2} .
$$

The output function $h$ and its corresponding output $y$ are then given by

$$
h(x, t)=|x-L i(t)|^{2}-\Phi^{2} \quad, \quad y=0 .
$$

We want to know whether, given the time signals $(u, i)$ and parameters $(L, \Phi)$, the particular solution $(\Psi, R)$ is the unique solution to System (10).

In the following, we answer this question depending on the properties of the particular solution $(\Psi, R)$. For that, we let $\theta$ be the unique time-signal such that

$$
\Psi(t)=L i(t)+\Phi\left(\begin{array}{c}
\cos (\theta(t)) \\
\sin (\theta(t))
\end{array}\right)
$$

and we denote

$$
z(t)=\left(\begin{array}{c}
\cos \theta(t) \\
\sin \theta(t)
\end{array}\right), i_{d q}(t)=I_{d q}(\theta(t)), \omega(t)=\dot{\theta}(t) .
$$

Our starting point is the following result.

\section{Theorem 1. If}

a) for all $t, \omega(t)=0$

or

b) there exists $t$ such that $\omega(t) \neq 0$, but for all $t, i_{d}(t)=0$, and $\frac{\omega}{i_{q}}(t)$ is defined and constant, then there exists an infinite number of solutions to System (10).

Otherwise, there exist at most 6 solutions.

Proof. Consider a solution $\left(x, x_{3}\right)$ to System (10). $x$ is necessarily of the form

with

$$
x(t)=x_{0}+\int_{0}^{t} u(\tau) d \tau-x_{3} \int_{0}^{t} i(\tau) d \tau
$$

$$
\dot{x}_{0}=0 \quad, \quad \dot{x}_{3}=0,
$$

and finding $\left(x, x_{3}\right)$ is equivalent to finding $\left(x_{0}, x_{3}\right)$. From (11), it follows for all $t$

$$
\begin{aligned}
0 & =|x(t)-L i(t)|^{2}-\left|x_{0}-L i(0)\right|^{2} \\
& =\left[x(t)-x_{0}-L(i(t)-i(0))\right]^{\top}\left[x(t)+x_{0}-L(i(t)+i(0))\right] \\
& =\tilde{\eta}\left(x_{3}, t\right)^{\top}\left[2\left(x_{0}-L i(0)\right)+\tilde{\eta}\left(x_{3}, t\right)\right]
\end{aligned}
$$


where we have defined the following affine function of $x_{3}$

$$
\tilde{\eta}\left(x_{3}, t\right)=\int_{0}^{t} u(\tau) d \tau-x_{3} \int_{0}^{t} i(\tau) d \tau-L(i(t)-i(0)) .
$$

We deduce that for any time $t$,

$$
2 \tilde{\eta}\left(x_{3}, t\right)^{\top}\left(x_{0}-\operatorname{Li}(0)\right)=-\tilde{\eta}\left(x_{3}, t\right)^{\top} \tilde{\eta}\left(x_{3}, t\right)=-\left|\tilde{\eta}\left(x_{3}, t\right)\right|^{2} .
$$

Therefore, unless $x_{3}$ makes $\tilde{\eta}\left(x_{3}, t_{1}\right)$ and $\tilde{\eta}\left(x_{3}, t_{2}\right)$ colinear for any $\left(t_{1}, t_{2}\right)$, there exits at most one possible value of $x_{0}$ for each $x_{3}$.

The rest of the proof then consists in showing that:

1. for $x_{3}$ such that the function $t \mapsto \tilde{\eta}\left(x_{3}, t\right)$ is not constant, there exist couples $\left(t_{1}, t_{2}\right)$ such that $\tilde{\eta}\left(x_{3}, t_{1}\right)$ and $\tilde{\eta}\left(x_{3}, t_{2}\right)$ are not colinear. $x_{0}$ is then uniquely determined, as a rational fraction of $x_{3}$ with a numerator of degree 3 and a denominator of degree 2 , and is solution of $\left|x_{0}-\operatorname{Li}(0)\right|^{2}=\Phi^{2}$. This implies that $x_{3}$ must be the root of a polynomial of degree 6 . Therefore, there are at most 6 solutions $\left(x, x_{3}\right)$ such that the function $t \mapsto \tilde{\eta}\left(x_{3}, t\right)$ is not constant.

2. to the values of $x_{3}$ such that the function $t \mapsto \tilde{\eta}\left(x_{3}, t\right)$ is constant, is associated an infinite number of solutions $\left(x, x_{3}\right)$.

3. $x_{3}$ makes the function $t \mapsto \tilde{\eta}\left(x_{3}, t\right)$ constant if and only if it satisfies for all $t$

$$
\begin{aligned}
& \left(R-x_{3}\right) i_{d}(t)=0 \\
& \left(R-x_{3}\right) i_{q}(t)=-\omega(t) \Phi .
\end{aligned}
$$

This is in particular the case for $x_{3}=R$ when $\omega(t)=0$ for all $t$.

Let us start by Point 1 . Take $x_{3}$ such that $\tilde{\eta}\left(x_{3},.\right)$ is not constant. There exists $t_{1}$ such that $\tilde{\eta}\left(x_{3}, t_{1}\right) \neq 0$. Thus, for some $t_{2} \neq t_{1}, \tilde{\eta}\left(x_{3}, t_{2}\right)$ colinear to $\tilde{\eta}\left(x_{3}, t_{1}\right)$ implies that there exists $\lambda$ such that $\tilde{\eta}\left(x_{3}, t_{2}\right)=\lambda \tilde{\eta}\left(x_{3}, t_{1}\right)$. But then,we have

$$
2 \lambda \tilde{\eta}\left(x_{3}, t_{1}\right)^{\top}\left(x_{0}-L i(0)\right)=-\lambda^{2}\left|\tilde{\eta}\left(x_{3}, t_{1}\right)\right|^{2}=-\lambda\left|\tilde{\eta}\left(x_{3}, t_{1}\right)\right|^{2}
$$

and necessarily $\lambda=1$ or $\lambda=0$, i-e $\tilde{\eta}\left(x_{3}, t_{2}\right)=\tilde{\eta}\left(x_{3}, t_{1}\right)$ or $\tilde{\eta}\left(x_{3}, t_{2}\right)=0$. But, since $\tilde{\eta}\left(x_{3},.\right)$ is continuous and not constant, there exists $t_{2}$ such that $\tilde{\eta}\left(x_{3}, t_{2}\right) \neq \tilde{\eta}\left(x_{3}, t_{1}\right)$ and $\tilde{\eta}\left(x_{3}, t_{2}\right) \neq 0$. Actually, still by continuity, we can even say that there exist two intervals $I_{1}$ and $I_{2}$ such that for all $\left(t_{1}, t_{2}\right)$ in $I_{1} \times I_{2}$, we have $\tilde{\eta}\left(x_{3}, t_{1}\right) \neq 0, \tilde{\eta}\left(x_{3}, t_{2}\right) \neq 0$ and $\tilde{\eta}\left(x_{3}, t_{2}\right) \neq \tilde{\eta}\left(x_{3}, t_{1}\right)$, i-e such that $\tilde{\eta}\left(x_{3}, t_{1}\right)$ and $\tilde{\eta}\left(x_{3}, t_{2}\right)$ are not colinear. For each such couple $\left(t_{1}, t_{2}\right), x_{0}$ is uniquely determined by the value of $x_{3}$. Indeed, from (15) we have

$$
2\left(\begin{array}{c}
\tilde{\eta}\left(x_{3}, t_{1}\right)^{\top} \\
\tilde{\eta}\left(x_{3}, t_{2}\right)^{\top}
\end{array}\right)\left(x_{0}-L i(0)\right)=-\left(\begin{array}{c}
\left|\tilde{\eta}\left(x_{3}, t_{1}\right)\right|^{2} \\
\left|\tilde{\eta}\left(x_{3}, t_{2}\right)\right|^{2}
\end{array}\right)
$$


and denoting

$$
J=\left(\begin{array}{cc}
0 & 1 \\
-1 & 0
\end{array}\right)
$$

it can be checked that

$$
\operatorname{det}\left(\begin{array}{l}
\tilde{\eta}\left(x_{3}, t_{1}\right)^{\top} \\
\tilde{\eta}\left(x_{3}, t_{2}\right)^{\top}
\end{array}\right)=\tilde{\eta}\left(x_{3}, t_{1}\right)^{\top} J \tilde{\eta}\left(x_{3}, t_{2}\right) \neq 0
$$

and

$$
\left(\begin{array}{c}
\tilde{\eta}\left(x_{3}, t_{1}\right)^{\top} \\
\tilde{\eta}\left(x_{3}, t_{2}\right)^{\top}
\end{array}\right)^{-1}=\frac{-1}{\tilde{\eta}\left(x_{3}, t_{1}\right)^{\top} J \tilde{\eta}\left(x_{3}, t_{2}\right)} J\left(\tilde{\eta}\left(x_{3}, t_{1}\right), \tilde{\eta}\left(x_{3}, t_{2}\right)\right) J
$$

so that necessarily

$$
x_{0}=\operatorname{Li}(0)+\frac{1}{2} \frac{J\left(\tilde{\eta}\left(x_{3}, t_{1}\right), \tilde{\eta}\left(x_{3}, t_{2}\right)\right)}{\tilde{\eta}\left(x_{3}, t_{1}\right)^{\top} J \tilde{\eta}\left(x_{3}, t_{2}\right)}\left(\begin{array}{c}
\left|\tilde{\eta}\left(x_{3}, t_{2}\right)\right|^{2} \\
-\left|\tilde{\eta}\left(x_{3}, t_{1}\right)\right|^{2}
\end{array}\right) .
$$

Replacing this expression in the constraint

$$
\left|x_{0}-\operatorname{Li}(0)\right|^{2}-\Phi^{2}=0,
$$

we obtain a polynomial of degree 6 in $x_{3}$ for each couple $\left(t_{1}, t_{2}\right)$ in $I_{1} \times I_{2}$. In order to deduce that there are at most 6 solutions $x_{3}$ making $\tilde{\eta}\left(x_{3},.\right)$ not constant, we need to prove that at least one of these polynomials is not a constant. It is possible to show that the coefficient of highest degree is given by $I\left(t_{1}\right)^{2} I\left(t_{2}\right)^{2}\left(I\left(t_{1}\right)-I\left(t_{2}\right)\right)$ with $I(t)=\int_{0}^{t} i(\tau) d \tau$. If $I\left(t_{1}\right)=0$ for all $t_{1}$ in $I_{1}$, then $i=\overparen{T}$ is zero on $I_{1}$ which is excluded by assumption, thus there exists $t_{1}$ in $I_{1}$ such that $I\left(t_{1}\right) \neq 0$. Now assume $I\left(t_{2}\right)=I\left(t_{1}\right)$ or $I\left(t_{2}\right)=0$ for all $t_{2}$ in $I_{2}$. Again this means that $i$ is zero on $I_{2}$, which is impossible. We conlude that there exists $\left(t_{1}, t_{2}\right)$ in $I_{1} \times I_{2}$ such that the corresponding polynomial is "truly" of order 6 (i-e with a nonzero coefficient of order 6 ) and therefore, there are at most 6 solutions $x_{3}$ making $\tilde{\eta}\left(x_{3},.\right)$ not constant, and for each of these values, there is a unique corresponding $x_{0}$. This characterizes at most 6 solutions $\left(x, x_{3}\right)$.

Now, for Point 2., take $x_{3}$ such that $\tilde{\eta}\left(x_{3},.\right)$ is constant. Since $\tilde{\eta}\left(x_{3}, 0\right)=0, \tilde{\eta}\left(x_{3}, t\right)=0$ for all $t$. It follows that any $x_{0}$ verifying $\left|x_{0}-L i(0)\right|=\Phi$ is solution, and there exists an infinity of solutions associated to this value of $x_{3}$.

Finally, for Point 3., $x_{3}$ makes $t \mapsto \tilde{\eta}\left(x_{3}, t\right)$ constant if and only if for all $t$, differentiating with respect to time,

$$
u(t)-x_{3} i(t)-L \dot{i}(t)=0 .
$$

But differentiating (13) with respect to time, we also know that

$$
u(t)-R i(t)=L \dot{\hat{i}}(t)+\omega \Phi\left(\begin{array}{c}
-\sin (\theta(t)) \\
\cos (\theta(t))
\end{array}\right)
$$

so that, by combining the two equations and multiplying by $\mathcal{R}(-\theta(t))$, we get system (16).

We can thus distinguish the following cases: 
- if $\omega(t)=0$ for all $t$, the function $t \mapsto \tilde{\eta}(R, t)$ is constant and, from 2), there is an infinite number of solutions $\left(x, x_{3}\right)$.

- if there exists $t$ such that $\omega(t) \neq 0$, and for all $t, i_{d}(t)=0$ and $\frac{\omega(t)}{i_{q}(t)}$ is defined and constant, then $x_{3}=R+\frac{\omega \Phi}{i_{q}}$ is the only solution to System (16) for all $t$ and from 2) there is an infinity of solutions $\left(x, x_{3}\right)$.

- otherwise, there exist no solution to System (16). Therefore, $\tilde{\eta}\left(x_{3}, \cdot\right)$ cannot be constant and from 1$)$ there are at most 6 solutions $\left(x, x_{3}\right)$ to our observability problem.

The fact that the system is not observable when the rotating speed is zero is not surprising since it is well-known even when $R$ is known. But, in the usual case where $\omega$ and $i_{d}$ are not constantly zero, this result says that there exist at most six possible solutions $\left(x, x_{3}\right)$. To get more information about those roots, one could study in detail this polynomial of degree six obtained in the proof. But its expression is too complex. In the next section, we show that the study of a stronger differential observability gives more hindsight about those solutions.

\subsection{Differential observability of order 3}

We consider now the following more stringent observability question expressed using the notation (12) for the output:

How many solutions in $\left(x, x_{3}\right)$ may the following equation have

$$
\overline{\mathbf{H}}_{3}\left(x, x_{3}, t\right)=0=\left(\begin{array}{c}
h(x, t) \\
\stackrel{\cdot}{h(x, t)} \\
\overparen{\cdot .} \\
h(x, t)
\end{array}\right) ?
$$

If there is only one solution the system is said differentially observable of order 3. Of course, in the cases of non observability identified in Theorem 1, the answer is more than 1 . But we want to study in more details what happens in the other cases, in particular when there exists a time $t$ such that

$$
\omega(t) \neq 0 \quad \text { and } \quad i_{d}(t) \neq 0 .
$$

By differentiations in (11), we obtain

$$
\overline{\mathbf{H}}_{3}\left(x, x_{3}, t\right)=\left(\begin{array}{c}
|x-L i(t)|^{2}-\Phi^{2} \\
2 \eta\left(x_{3}, t\right)^{\top}(x-L i(t)) \\
2 \dot{\eta}\left(x_{3}, t\right)^{\top}(x-L i(t))+2\left|\eta\left(x_{3}, t\right)\right|^{2}
\end{array}\right)
$$

where we have denoted

$$
\eta\left(x_{3}, t\right)=u(t)-x_{3} i(t)-L \dot{i}(t)
$$


We have the following result:

Theorem 2. Consider a time $t$ such that $\omega(t) \neq 0$ and $i_{d}(t) \neq 0$. There are as many solutions $\left(x, x_{3}\right)$ to the equation

$$
\overline{\mathbf{H}}_{3}\left(x, x_{3}, t\right)=0,
$$

as the number of distinct real roots of the following polynomial of degree six:

$$
\begin{aligned}
P\left(x_{3}, t\right)=\omega(t)^{6} \Phi^{6}\left[\left(1-\frac{\left(R-x_{3}\right)}{\omega(t) \Phi}\left(\overparen{\left(\frac{i_{d}}{\omega}\right)}(t)-2 i_{q}(t)\right)+\frac{\left(R-x_{3}\right)^{2}}{\omega(t)^{2} \Phi^{2}} \mu(t)|i(t)|^{2}\right)^{2}\right. \\
\left.-\left(1+\frac{\left(R-x_{3}\right)}{\omega(t) \Phi} 2 i_{q}+\frac{\left(R-x_{3}\right)^{2}}{\omega(t)^{2} \Phi^{2}}|i(t)|^{2}\right)^{3}\right]
\end{aligned}
$$

where ${ }^{1} \mu(t)=\frac{1}{\omega(t)} \frac{\left[i(t)^{\top} J \dot{i(t)}\right]}{|i(t)|^{2}}$. If besides $i_{q}(t) \neq-\overbrace{\left(\frac{i_{d}}{\omega}\right)}(t)$, then $P\left(x_{3}, t\right)$ admits at least two distinct real roots.

Proof. It is interesting to note that $\eta\left(x_{3}, t\right)=\widetilde{\tilde{\eta}\left(x_{3}, t\right)}$, where $\tilde{\eta}$ is defined in (14), so that what is done in this proof is somehow the differential version of the proof of Theorem 1 . To study how many solutions in $\left(x, x_{3}\right)$ the equation (17) has, we remark that the second and third component of $\overline{\mathbf{H}}_{3}$ give a linear system in $x-L i$. So our approach is to solve this system and replace it in the first component. This gives a function of $x_{3}$ only. Hence the first question is invertibility of the linear system, i.e. colinearity of $\eta\left(x_{3}, t\right)$ and $\dot{\eta}\left(x_{3}, t\right)$.

Assume that, for $t$ given in the statement and some $x_{3}, \eta\left(x_{3}, t\right)$ is non zero and colinear with $\dot{\eta}\left(x_{3}, t\right)$, namely $\dot{\eta}\left(x_{3}, t\right)=\lambda \eta\left(x_{3}, t\right)$. Then, (17) gives

$$
\eta\left(x_{3}, t\right)^{\top}(x-L i(t))=0, \lambda \eta\left(x_{3}, t\right)^{\top}(x-L i(t))=-\left|\eta\left(x_{3}, t\right)\right|^{2}
$$

and necessarily $\eta\left(x_{3}, t\right)=0$ which is a contradiction. Therefore, colinearity can only happen if $\eta\left(x_{3}, t\right)=0$. But differentiating (13) with respect to time and combining this expression with (18), we get

$$
\eta\left(x_{3}, t\right)=-\omega(t) \Phi J z(t)+\left(R-x_{3}\right) i(t) .
$$

By imposing $\eta\left(x_{3}, t\right)$ to be zero and multiplying by $\mathcal{R}(-\theta)$, we obtain $\left(R-x_{3}\right) i_{d}(t)=0$ and $\left(R-x_{3}\right) i_{q}(t)=-\omega(t) \Phi$, which is impossible since $\omega(t) \neq 0$ and $i_{d}(t) \neq 0$. Therefore, for all $x_{3}$, $\eta\left(x_{3}, t\right)$ and $\dot{\eta}\left(x_{3}, t\right)$ are not colinear.

It follows that we can get $x$ from the second and third components of $\overline{\mathbf{H}}_{3}$, namely

$$
\begin{aligned}
(x-L i(t))^{\top} \eta\left(x_{3}, t\right) & =0 \\
(x-L i(t))^{\top} \dot{\eta}\left(x_{3}, t\right) & =-\left|\eta\left(x_{3}, t\right)\right|^{2}
\end{aligned}
$$

\footnotetext{
${ }^{1} \mu$ is the ratio between $\omega$ and the rotation speed of $i$.
} 
i.e.

$$
x-L i(t)=\frac{\left|\eta\left(x_{3}, t\right)\right|^{2}}{\eta\left(x_{3}, t\right)^{\top} J \dot{\eta}\left(x_{3}, t\right)} J \eta\left(x_{3}, t\right) .
$$

Inserting this expression in the first component of $\overline{\mathbf{H}}_{3}$ gives

$$
\begin{aligned}
\Phi^{2} & =\left|\frac{\left|\eta\left(x_{3}, t\right)\right|^{2}}{\eta\left(x_{3}, t\right)^{\top} J \dot{\eta}\left(x_{3}, t\right)} J \eta\left(x_{3}, t\right)\right|^{2} \\
& =\frac{\left|\eta\left(x_{3}, t\right)\right|^{6}}{\left[\eta\left(x_{3}, t\right)^{\top} J \dot{\eta}\left(x_{3}, t\right)\right]^{2}}
\end{aligned}
$$

so that $x_{3}$ is a root of the following polynomial

$$
P\left(x_{3}, t\right)=\Phi^{2}\left[\eta\left(x_{3}, t\right)^{\top} J \dot{\eta}\left(x_{3}, t\right)\right]^{2}-\left|\eta\left(x_{3}, t\right)\right|^{6} .
$$

Differentiating (20), we get

$$
\dot{\eta}\left(x_{3}, t\right)=-\dot{\omega}(t) \Phi J z(t)-\omega(t)^{2} \Phi z(t)+\left(R-x_{3}\right) \dot{\hat{i}}(t)
$$

which yields

$$
\begin{aligned}
\eta\left(x_{3}, t\right)^{\top} J \dot{\eta}\left(x_{3}, t\right) & =\operatorname{det}\left(\eta\left(x_{3}, t\right), \dot{\eta}\left(x_{3}, t\right)\right) \\
& =\omega^{3} \Phi^{2}-\left(R-x_{3}\right) \Phi\left[\omega^{2} i^{\top} J z-\dot{\omega} i^{\top} z+\omega \dot{\overparen{i}}^{\top} z\right]+\left(R-x_{3}\right)^{2} i^{\top} J \dot{\hat{i}} \\
& =\omega^{3} \Phi^{2}-\left(R-x_{3}\right) \Phi \omega^{2}\left[-2 i_{q}+{\dot{\left(\frac{i_{d}}{\omega}\right)}}^{\top}\right]+\left(R-x_{3}\right)^{2} i^{\top} \dot{\widehat{i}}
\end{aligned}
$$

where we have used the fact that $i^{\top} z=i_{d}$ and $i^{\top} J z=-i_{q}$. Inserting those expressions in the expression of $P$, we get the polynomial (19). The coefficient of degree 6 is $|i|^{6}$ which is non zero by assumption. Since the value of $x$ is imposed by that of $x_{3}$, we get the first statement.

$R$ is one real root of $P\left(x_{3}, t\right)$. Because $P$ has an even degree, there is at least another real root. But we have $\frac{\partial P}{\partial x_{3}}(R, t)=0$ iff $i_{q}(t)=-\overbrace{\left(\frac{i_{d}}{\omega}\right)}(t)$. Therefore, if this condition is not satisfied, $R$ is a simple root and there exist at least two distinct real roots.

With this result, we recover the fact that there exist at most 6 possible values for $\left(x, x_{3}\right)$. Also, because $i_{q}(t)=-\overparen{\left(\frac{i_{d}}{\omega}\right)}(t)$ is likely to happen for specific inputs only, we can expect the system to be generally not differentially observable, with at least two candidates for $\left(x, x_{3}\right)$ at each time $t$. We may wonder if $P$ admits other real roots, or if its other 4 roots are complex. To answer this question, we restrict our attention to the particular case where $\omega, i_{d}$ and $i_{q}$ are constant. 


\subsection{Particular case where $\omega, i_{d}$ and $i_{q}$ are constant}

In this case, we already see from Theorem 2 that differential observability is possible only if $i_{q}=0$. Let us describe more precisely the roots of $P$.

Corollary 1. If $\omega, i_{d}$ and $i_{q}$ are constant with $\omega \neq 0$ and $i_{d} \neq 0$, then the polynomial $P$ in (19) has only two roots in $x_{3}$ given by

$$
x_{3}=R \quad, \quad x_{3}=R+\frac{2 \Phi \omega i_{q}}{|i|^{2}} .
$$

Therefore, the equation $\overline{\mathbf{H}}_{3}\left(x, x_{3}, t\right)=0$ admits one solution if $i_{q}=0$ and two distinct solutions if $i_{q} \neq 0$.

Proof. We have:

$$
i^{\top} J \stackrel{\grave{i}}{=} i_{q d}^{\top} \mathcal{R}(-\theta) \mathcal{R}\left(-\frac{\pi}{2}\right) \overparen{\mathcal{R}(-\theta) i_{d q}}
$$

So when $i_{d q}$ is constant, we obtain:

$$
\begin{aligned}
& i^{\top} J \dot{\widehat{i}}=i_{d q}^{\top} \mathcal{R}(-\theta) \mathcal{R}\left(-\frac{\pi}{2}\right) \overparen{\mathcal{R}(-\theta)} i_{d q} \\
& =\omega i_{d q}^{\top} \mathcal{R}(-\theta) \mathcal{R}\left(-\frac{\pi}{2}\right) \mathcal{R}\left(\frac{\pi}{2}\right) \mathcal{R}(\theta) i_{d q} \\
& =\omega\left|i_{d q}\right|^{2}=\omega|i|^{2} \text {. }
\end{aligned}
$$

This implies $\mu(t)=1$ and

$$
P\left(x_{3}\right)=-\omega^{6} \Phi^{6}\left(1+\frac{\left(R-x_{3}\right)}{\omega \Phi} 2 i_{q}+\frac{\left(R-x_{3}\right)^{2}}{\omega^{2} \Phi^{2}}|i|^{2}\right)^{2} \frac{\left(R-x_{3}\right)}{\omega \Phi}\left(2 i_{q}+\frac{\left(R-x_{3}\right)}{\omega \Phi}|i|^{2}\right) .
$$

The polynomial $1+2 i_{q} X+X^{2}|i|^{2}$ has a discriminant equal to $-4 i_{d}^{2}<0$ and does not admit any real root. The conclusion follows. Note that in this case, according to (24), $P$ also writes

$$
P\left(x_{3}\right)=-\Phi^{2} \operatorname{det}\left(\eta\left(x_{3}\right), \dot{\eta}\left(x_{3}\right)\right)^{2} \times \frac{\left(R-x_{3}\right)}{\omega \Phi}\left(2 i_{q}+\frac{\left(R-x_{3}\right)}{\omega \Phi}|i|^{2}\right) .
$$

This theorem says that the system is not differentially observable of order 3 unless $i_{q}=0$. This does not mean that the system is not observable because the solution corresponding to $x_{3}=$ $R+\frac{2 \Phi \omega i_{q}}{|i|^{2}}$ may not be admissible for System (10). Actually, it turns out that both solutions are truly indistinguishable:

Theorem 3. Assume $\omega, i_{d}$ and $i_{q}$ are constants such that $\omega \neq 0$ and $i_{d} \neq 0$. There exist exactly two indistinguishable solutions $\left(x, x_{3}\right)$ to System $(10)$. They are of the form $(\Psi, R)$ and $\left(\Psi_{\delta}, R_{\delta}\right)$ 
with

$$
\begin{gathered}
R_{\delta}=R+\frac{2 \Phi \omega i_{q}}{|i|^{2}}, \quad i_{d, \delta}=i_{d} \quad, \quad i_{q, \delta}=-i_{q}, \\
\omega_{\delta}=\dot{\theta}_{\delta}=\dot{\theta}=\omega, \\
\Psi_{\delta}(t)=L i(t)+\frac{\left|\eta\left(R_{\delta}, t\right)\right|^{2}}{\eta\left(R_{\delta}, t\right)^{\top} J \dot{\eta}\left(R_{\delta}, t\right)} J \eta\left(R_{\delta}, t\right),
\end{gathered}
$$

with $\eta$ defined in (18), $\theta_{\delta}=\Theta\left(\Psi_{\delta}\right)$ and $i_{d q, \delta}=I_{d q}\left(\theta_{\delta}\right)$. Besides, if $(\hat{R}, \hat{\theta})$ is one of the solutions $(R, \theta)$ or $\left(R_{\delta}, \theta_{\delta}\right)$, then the other solution is

$$
\left(\hat{R}+\frac{2 \Phi \hat{\omega} \hat{i_{q}}}{|i|^{2}}, \hat{\theta}+\arctan _{2}\left(2 \frac{\hat{i_{q} i_{d}}}{|i|^{2}}, 1-2 \frac{\hat{i}^{2}}{|i|^{2}}\right)\right)
$$

where $\hat{i_{d q}}=I_{d q}(\hat{\theta})$ and $\hat{\omega}=\dot{\hat{\theta}}$.

Proof. See Appendix .1.

We conclude that the system is not observable if $i_{q} \neq 0$, but with only two possible solutions. It was observed in [18] that they actually correspond to those recently exhibited in [17] in the context of induction motors, once we apply the letter swap proposed in [12] to go from an induction motor model to a PMSM one. Those two indistinguishable trajectories can be dissociated with the additional information of the sign of $i_{q}$. In fact, the sign of $i_{q}$ determines the mode of use of the machine: if $i_{q}>0$, the torque is positive and the machine acts as a motor, whereas if $i_{q}<0$, the torque is negative and the machine acts as a generator. In other words, both solutions can be distinguished if we know the mode of use of the motor.

This result also says that, in practice, if an estimation $\hat{R}$ among $\left\{R, R_{\delta}\right\}$ is available (for instance thanks to an observer), it is possible to find the other candidate. However for this, we need as estimation of the rotation speed $\omega$. This will be exploited in the estimation algorithm presented below.

Remark 1. In fact, from a physical point of view, those two values of $R$ correspond to two systems with same total energy but with different energy repartition. Indeed, the total energy is

$$
E=\frac{L}{2}|i|^{2}+\frac{\mathcal{I}}{2} \omega_{r}^{2}
$$

From (3) and the fact that $\omega=n_{p} \omega_{r}$, it satisfies:

$$
\dot{E}=-R|i|^{2}+u^{\top} i-\frac{\omega}{n_{p}} \tau_{L}
$$

When $i_{d}, i_{q}$ and $\omega$ are constant, $E$ is constant and we have:

$$
-R|i|^{2}+u^{\top} i-\frac{1}{n_{p}} \omega \tau_{L}=0 \quad, \quad \Phi n_{p} i_{q}=-\Phi n_{p} i^{\top} J z=\tau_{L}
$$


So when $u, i$ and $\omega$ are known but the sign of the mechanical power $\frac{\omega}{n_{p}} \tau_{L}=\Phi i_{q} \omega$ is unknown, there are two possible values for $R$

$$
R=\frac{u^{\top} i-\Phi\left|i_{q}\right||\omega|}{|i|^{2}} \text { or } \frac{u^{\top} i+\Phi\left|i_{q}\right||\omega|}{|i|^{2}}
$$

The difference $2 \frac{\Phi\left|i_{q}\right||\omega|}{|i|^{2}}$ between the two values is exactly what we have found above. And the fact that $\frac{u^{\top} i}{|i|^{2}}$ is larger or smaller than $R$ depends only the sign the mechanical power.

We conclude from this observability analysis that System (10) is not observable when $\omega$ or $i_{d}$ remains at 0 . However, when $\omega$ and $i_{d}$ are nonzero, the number of indistinguishable trajectories is reduced to maximum six: the possible values of $R$ are the roots of a polynomial $P$ of degree six given by (19). This polynomial is likely to have at least two real roots in the general case, and only two if $\omega, i_{d}$ and $i_{q}$ are constant. In that latter case, there are exactly two indistinguishable trajectories and they can be distinguished with the additional information of the sign of $i_{q}$. Note that by continuity, if $\omega, i_{d}$ and $i_{q}$ vary slowly, we may expect $P$ to have only two real roots at each time $t$.

\section{From solving a single equation at different times to several equa- tions at the same time}

With the observability study in hand, we would like to design an estimation algorithm. For this, we follow the Luenberger methodology. It consists in finding a transformation $\left(x, x_{3}, t\right) \mapsto T\left(x, x_{3}, t\right)$ that maps the system dynamics (10) into a Hurwitz form

$$
\dot{z}=A z+B(y)
$$

of a certain dimension with $A$ a Hurwitz matrix. Indeed, implementing

$$
\dot{\hat{z}}=A \hat{z}+B(y)
$$

for any initial conditions then gives an estimate $\hat{z}$ which asymptotically converges to $z=T\left(x, x_{3}, t\right)$. If $\left(x, x_{3}\right) \mapsto T\left(x, x_{3}, t\right)$ is besides injective (uniformly in time $t$ ), it is possible to deduce an estimate $\left(\hat{x}, \hat{x}_{3}\right)$ of $\left(x, x_{3}\right)$ by inverting the transformation. Although the injectivity of $T$ typically requires the observability of the system [24], this design may still be fruitful in the context of non observable systems like the PMSM under study here.

In fact, in the present context, the output map defined in (12) remains zero at all times. Therefore, using this output map in the Luenberger design and $B$ linear gives $\dot{z}=A z$. It follows that a possible estimate is simply $\hat{z}=0$, which becomes valid exponentially fast, and the Luenberger design comes back to solving online $T\left(\hat{x}(t), \hat{x}_{3}(t), t\right)=0$ at all times. More precisely, choosing $A$ 
diagonal leads us to take $T=\left(T_{\lambda_{1}}, \cdots, T_{\lambda_{m}}\right)$ for some complex numbers $\lambda_{1}, \ldots, \lambda_{m}$ with positive real part, and $T_{\lambda}: \mathbb{R}^{2} \times \mathbb{R}^{+} \times \mathbb{R} \rightarrow \mathbb{R}$ of the form

$$
T_{\lambda}\left(x, x_{3}, t\right)=\lambda^{2} x^{\top} x+\lambda c_{\lambda}(t)^{\top} x+\lambda x_{3} b_{\lambda}(t)^{\top} x+a_{\lambda}(t) x_{3}+d_{\lambda}(t) x_{3}^{2}-e_{\lambda}
$$

with $a_{\lambda}, b_{\lambda}, c_{\lambda}, d_{\lambda}$, and $e_{\lambda}$ the outputs of the following filters

$$
\begin{aligned}
& \dot{a_{\lambda}}=-\lambda\left(a_{\lambda}-c_{\lambda}^{\top} i+b_{\lambda}^{\top} u\right) \\
& \dot{b_{\lambda}}=-\lambda\left(b_{\lambda}-2 i\right) \\
& \dot{c_{\lambda}}=-\lambda\left(c_{\lambda}+2 u+2 \lambda L i\right) \\
& \dot{d_{\lambda}}=-\lambda\left(d_{\lambda}-b_{\lambda}^{\top} i\right) \\
& \dot{e_{\lambda}}=-\lambda\left(e_{\lambda}-c_{\lambda}^{\top} u+\lambda^{2} L^{2}|i|^{2}-\lambda^{2} \Phi^{2}\right) .
\end{aligned}
$$

We have the following result.

Lemma 1. For any complex number $\lambda$ with strictly positive real part, for any initial conditions in the filters (30), any solution $(\Psi, R)$ to System (10) verifies

$$
T_{\lambda}(\Psi(t), R, t)=\exp (-\lambda t) T_{\lambda}(\Psi(0), R, 0) .
$$

Proof. Straightforward computations show that the function $t \rightarrow T_{\lambda}(\Psi(t), R, t)$ is solution to $\dot{z}=$ $-\lambda z$, hence the result.

This means that, up to neglecting terms decaying with time $t$ as $\exp (-\lambda t)$, we can replace the single measurement equation

$$
\Phi^{2}-L|i(t)|^{2}=x^{\top} x-2 \operatorname{Li}(t)^{\top} x
$$

exact at each time $t$, by as many following equations as the number of $\lambda$ we choose to pick

$$
T_{\lambda}\left(x, x_{3}, t\right)=0
$$

which (exponentially fast) become exact as $t$ goes to infinity.

This leads to the following question: can we choose some number $m$ of $\lambda_{i}$, such that, given $t$, $\left(x, x_{3}\right) \mapsto T\left(x, x_{3}, t\right)=\left(T_{\lambda_{1}}\left(x, x_{3}, t\right), \ldots, T_{\lambda_{m}}\left(x, x_{3}, t\right)\right)$ is injective? Indeed, in this case, along any solution we would have for this $t$, the implication

$$
T\left(x, x_{3}, t\right)=T(\Psi, R, t) \quad \Longrightarrow \quad x=\Psi, \quad x_{3}=R .
$$

Unfortunately, as we have established, when $\omega, i_{d}$ and $i_{q}$ are constant, two solutions $(\Psi, R)$ and $\left(\Psi_{\delta}, R_{\delta}\right)$ are not distinguishable by the dynamics, and thus necessarily, whatever $m$ is, we have

$$
T(\Psi(t), R, t)=T\left(\Psi_{\delta}(t), R_{\delta}, t\right) \quad \forall t .
$$

This means that it is hopeless to prove the injectivity of $T$, but it may still be possible to recover the (at least two and at most six) possible values of $(\Psi, R)$. 


\subsection{Inversion of $T$}

As observed in $[22,23]$, there usually is a tight link between the Luenberger map $T$ of dimension $m$ and the map $\overline{\mathbf{H}}_{m}$ made of the output map and its $m-1$ derivatives. Inspired by the study of differential observability in Section 2 , we therefore select $m=3$ and take $\lambda_{1}, \lambda_{2}, \lambda_{3}$ as positive real numbers to simplify the notations. We could as well pick one real number and one complex number.

We denote:

$$
\begin{aligned}
T\left(x, x_{3}, t\right) & =\left(\begin{array}{c}
T_{\lambda_{1}}\left(x, x_{3}, t\right) \\
T_{\lambda_{2}}\left(x, x_{3}, t\right) \\
T_{\lambda_{3}}\left(x, x_{3}, t\right)
\end{array}\right) \\
& =m_{\lambda} x^{\top} x+\Lambda\left(c(t)+x_{3} b(t)\right) x+a(t) x_{3}+d(t) x_{3}^{2}-e(t)
\end{aligned}
$$

where

$$
\begin{gathered}
\Lambda=\left(\begin{array}{ccc}
\lambda_{1} & 0 & 0 \\
0 & \lambda_{2} & 0 \\
0 & 0 & \lambda_{3}
\end{array}\right) \\
m_{\lambda}=\left(\begin{array}{c}
\lambda_{1}^{2} \\
\lambda_{2}^{2} \\
\lambda_{3}^{2}
\end{array}\right) \quad, \quad a=\left(\begin{array}{c}
a_{\lambda_{1}} \\
a_{\lambda_{2}} \\
a_{\lambda_{3}}
\end{array}\right) \quad, \quad b=\left(\begin{array}{c}
b_{\lambda_{1}}^{\top} \\
b_{\lambda_{2}}^{\top} \\
b_{\lambda_{3}}^{\top}
\end{array}\right) \\
c=\left(\begin{array}{c}
c_{\lambda_{1}}^{\top} \\
c_{\lambda_{2}}^{\top} \\
c_{\lambda_{3}}^{\top}
\end{array}\right) \quad, \quad d=\left(\begin{array}{c}
d_{\lambda_{1}} \\
d_{\lambda_{2}} \\
d_{\lambda_{3}}
\end{array}\right) \quad, \quad e=\left(\begin{array}{c}
e_{\lambda_{1}} \\
e_{\lambda_{2}} \\
e_{\lambda_{3}}
\end{array}\right)
\end{gathered}
$$

Because of Lemma 1, we are interested in finding the solutions $\left(x, x_{3}\right)$ to:

$$
T\left(x, x_{3}, t\right)=0
$$

at each time $t$. To compact the notations, we let

$$
M_{\lambda}=\left(\begin{array}{ccc}
\lambda_{2}^{2} & -\lambda_{1}^{2} & 0 \\
0 & \lambda_{3}^{2} & -\lambda_{2}^{2}
\end{array}\right)
$$

which is such that

$$
M_{\lambda} m_{\lambda}=0,
$$

and introduce the following functions of $\left(x_{3}, t\right)$

$$
\begin{aligned}
\mathcal{M}\left(x_{3}, t\right) & =M_{\lambda} \Lambda\left(c(t)+x_{3} b(t)\right) \\
\chi\left(x_{3}, t\right) & =\mathcal{M}\left(x_{3}, t\right)^{-1} M_{\lambda}\left(e(t)-a(t) x_{3}-d(t) x_{3}^{2}\right) \\
J\left(x_{3}, t\right) & =m_{\lambda}^{\top}\left(e(t)-T\left(\chi\left(x_{3}, t\right), x_{3}, t\right)\right) .
\end{aligned}
$$


For $\left(x, x_{3}, t\right)$ verifying $(32)$ and such that $\mathcal{M}\left(x_{3}, t\right)$ is invertible, we have

$$
J\left(x_{3}, t\right)=0 \quad, \quad x=\chi\left(x_{3}, t\right)
$$

i.e., the roots of $J(\cdot, t)$ give the possible values of $x_{3}$ and $\chi\left(x_{3}, t\right)$ the corresponding value of $x$.

In fact, for all times $t$, except maybe for the unlikely ones where both $\operatorname{det}\left(M_{\lambda} \Lambda c(t)\right)$ and $\operatorname{det}\left(M_{\lambda} \Lambda b(t)\right)$ are zero, the determinant of the $2 \times 2$-matrix $\mathcal{M}\left(x_{3}, t\right)$, affine in $x_{3}$, is a polynomial of degree 2 in $x_{3}$. Therefore, $\mathcal{M}\left(x_{3}, t\right)$ is invertible for all $x_{3}$ except maybe for at most two bad values denoted $\left\{x_{3 a}(t), x_{3 b}(t)\right\}$. Then, $\chi\left(x_{3}, t\right)$ is a two-dimensional matrix made of rational fractions in $x_{3}$ with numerator of degree 3 and denominator of degree 2 , defined everywhere except at $\left\{x_{3 a}(t), x_{3 b}(t)\right\}$. Similarly $J\left(x_{3}, t\right)$ is a rational fraction in $x_{3}$, with numerator of degree 6 and denominator of degree 4 defined everywhere except maybe at $\left\{x_{3 a}(t), x_{3 b}(t)\right\}$. We have the following result.

Theorem 4. Consider any $\left(\lambda_{1}, \lambda_{2}, \lambda_{3}\right)$ in $\left(\mathbb{R}_{>0}\right)^{3}$, any initial conditions of the filters (30). Assume the input $(u, i)$ is bounded. Then, any solution $(\Psi, R)$ to System (10) such that there exist positive real numbers $\bar{t}$ and $\underline{\delta}$ such that

$$
|\operatorname{det}(\mathcal{M}(R, t))| \geq \underline{\delta}>0 \quad \forall t \geq \bar{t}
$$

verifies

$$
\lim _{t \rightarrow+\infty} \Psi(t)-\chi(R, t)=0 \quad, \quad \lim _{t \rightarrow+\infty} J(R, t)=0 .
$$

Proof. See Appendix .2.

This result says that, if $\mathcal{M}(R, t)$ is invertible for all $t$, our model is equivalent, at least asymptotically (and exponentially fast) to

$$
\left\{\begin{aligned}
0 & =J(R, t) \\
\Psi & =\chi(R, t)
\end{aligned}\right.
$$

We have thus replaced the scalar equation $y(t)=h(\Psi(t), t)$, written with the compact notation (12), valid at all times $t$, by the three equations in (35) to be solved at each time $t$. Because $\Psi$ is explicitly given by the last two equations, the problem of estimating $(\Psi, R)$ reduces to finding the roots of $x_{3} \mapsto J\left(x_{3}, t\right)$ at each time $t$.

In order to deduce from this a possible estimation algorithm, we must therefore answer the following two questions:

- Is the matrix $\mathcal{M}(R, t)$, involved in the expression of $\chi(R, t)$, invertible, or, more precisely, is $|\operatorname{det}(\mathcal{M}(R, t))|$ lower-bounded at least after a certain time?

- If yes, is $R$ the only root of $J(\cdot, t)$ (on its domain of definition) at least after a certain time? If not, which are the other solutions?

It turns out that the observability analysis carried out above gives some insight about those questions. 


\subsection{Link with observability}

The following technical lemma shows that there is a tight link between $\mathcal{M}\left(x_{3}, t\right)$ and $J$ on one side and $\left(\eta\left(x_{3}, t\right), \dot{\eta}\left(x_{3}, t\right)\right)$ and $P$ encountered during the observability study on the other.

Lemma 2. Assume the inputs $(u, i)$ and their time derivatives are bounded. We have the following relations

$$
\operatorname{det}\left(\mathcal{M}\left(x_{3}, t\right)\right)=O\left(\lambda^{5}\right) \operatorname{det}\left(\eta\left(x_{3}, t\right), \dot{\eta}\left(x_{3}, t\right)\right)+O\left(\lambda^{4}\right),
$$

and if $\left(x_{3}, t\right)$ is such that the matrices $\mathcal{M}\left(x_{3}, t\right)$ and $\left(\eta\left(x_{3}, t\right), \dot{\eta}\left(x_{3}, t\right)\right)$ are invertible

$$
\begin{gathered}
\left(\begin{array}{c}
\eta\left(x_{3}, t\right)^{\top} \\
\dot{\eta}\left(x_{3}, t\right)^{\top}
\end{array}\right)\left(\chi\left(x_{3}, t\right)-L i\right)=\left(\begin{array}{c}
0 \\
-\mid \eta\left(x_{3},\left.t\right|^{2}\right.
\end{array}\right)+O\left(\frac{1}{\lambda}\right) \\
J\left(x_{3}, t\right)=O\left(\lambda^{4}\right) \frac{P\left(x_{3}, t\right)}{\operatorname{det}\left(\eta\left(x_{3}, t\right), \dot{\eta}\left(x_{3}, t\right)\right)^{2}}+O\left(\lambda^{3}\right)
\end{gathered}
$$

with $\eta$ defined in (18), $P$ in (19), and the notation $O\left(\lambda^{k}\right)$ indicates a term $f\left(\lambda_{1}, \lambda_{2}, \lambda_{3}, x_{3}, t\right)$ such that, for any fixed $\left(\lambda_{1}, \lambda_{2}, \lambda_{3}, x_{3}\right), t \mapsto f\left(\lambda_{1}, \lambda_{2}, \lambda_{3}, x_{3}, t\right)$ is bounded and for any $t \geq 0$,

$$
\limsup _{\alpha \rightarrow+\infty}\left|\frac{f\left(\alpha \lambda_{1}, \alpha \lambda_{2}, \alpha \lambda_{3}, x_{3}, t\right)}{\alpha^{k}}\right|<+\infty \text {. }
$$

Proof. This is obtained by expanding the filter solutions as (positive or negative) powers of $\lambda$. See Appendix .3.

It follows that, when the $\lambda_{i}$ are sufficiently large, $\mathcal{M}$ is tightly related to $\left(\eta\left(x_{3}, t\right), \dot{\eta}\left(x_{3}, t\right)\right)$ and $J$ is tightly related to $P$. We can thus hope to transfer the known properties of those functions to $\mathcal{M}$ and $J$.

\subsubsection{About Equation (36)}

From (36), we get the impression that the invertibility of $\mathcal{M}\left(x_{3}, t\right)$ is related to that of $\left(\eta\left(x_{3}, t\right), \dot{\eta}\left(x_{3}, t\right)\right)$, at least for $\lambda_{i}$ sufficiently large. Actually, we have a more precise result.

Theorem 5. Assume the inputs $(u, i)$ and their derivatives are bounded. Pick three distinct positive real numbers $\left(\tilde{\lambda}_{1}, \tilde{\lambda}_{2}, \tilde{\lambda}_{3}\right)$ and select the $\lambda_{i}$ in the observer as

$$
\left(\lambda_{1}, \lambda_{2}, \lambda_{3}\right)=\left(\alpha \tilde{\lambda}_{1}, \alpha \tilde{\lambda}_{2}, \alpha \tilde{\lambda}_{3}\right)
$$

with $\alpha$ a positive real number to be tuned. Then, for any initial conditions in the filters, there exists $\bar{t}: \mathbb{R}^{+} \rightarrow \mathbb{R}^{+}$satisfying

$$
\lim _{\alpha \rightarrow+\infty} \bar{t}(\alpha)=0
$$

and, 
1.) for each $x_{3}$ and each $\underline{d}$, satisfying for all $t$,

$$
\left|\operatorname{det}\left(\eta\left(x_{3}, t\right), \dot{\eta}\left(x_{3}, t\right)\right)\right| \geq \underline{d}>0
$$

there exists $\underline{\alpha}>0$ and $\underline{\delta}>0$, such that for any $\alpha \geq \underline{\alpha}$,

$$
\left|\operatorname{det}\left(\mathcal{M}\left(x_{3}, t\right)\right)\right| \geq \underline{\delta} \quad \forall t \geq \bar{t}(\alpha)
$$

2.) If there exists $\underline{\omega}>0$ such that $|\omega(t)| \geq \underline{\omega}$ for all $t$, there exists $\underline{\alpha}>0$ and $\underline{\delta}>0$ such that, for any $\alpha \geq \underline{\alpha}$,

$$
|\operatorname{det}(\mathcal{M}(R, t))| \geq \underline{\delta} \quad \forall t \geq \bar{t}(\alpha) .
$$

In particular, if $\omega, i_{d}$ and $i_{q}$ are constant with $\omega \neq 0$ and $i_{d} \neq 0$, then, for any $0<\varepsilon<1$, there exists $\underline{\alpha}>0$ such that for all $\alpha \geq \underline{\alpha}$ and for all $t \geq \bar{t}(\alpha)$, the only two roots of $\operatorname{det}\left(\mathcal{M}\left(x_{3}, t\right)\right)$ are complex and located in the annulus ${ }^{2} C\left(R, \underline{r_{\varepsilon}}, \overline{r_{\varepsilon}}\right)$ with

$$
\underline{r_{\varepsilon}}=\frac{\omega \Phi}{|i|}(1-\varepsilon) \quad, \quad \overline{r_{\varepsilon}}=\frac{\omega \Phi}{|i|}(1+\varepsilon)
$$

Proof. This is done by bounding the terms $O\left(\lambda^{k}\right)$ in Lemma 2 uniformly in time, and applying Rouché's theorem. See Appendix .4.

We conclude that, if $\omega$ is lower-bounded away from zero, it is possible to guarantee the invertibility of $\mathcal{M}(R, t)$ at least after a certain time $\bar{t}$ by taking the $\lambda_{i}$ sufficiently large. Also, the larger the $\lambda_{i}$, the shorter the time needed before $\mathcal{M}(R, t)$ becomes invertible. Therefore, at least after some time, any value of $x_{3}$ making $\mathcal{M}\left(x_{3}, t\right)$ non invertible (and thus $J\left(x_{3}, t\right)$ not defined) cannot be $R$ and can be put aside in the algorithm. Note that according to Theorem 5 , if $\omega, i_{d}$ and $i_{q}$ are constant with $\omega \neq 0$ and $i_{d} \neq 0, \mathcal{M}\left(x_{3}, t\right)$ becomes invertible for all $x_{3}$ in $\mathbb{R}$ for all $t \geq \bar{t}$ and $J\left(x_{3}, t\right)$ is then defined also for all $x_{3}$ in $\mathbb{R}$.

\subsubsection{About Equation (37)}

(37) implies that $\chi\left(x_{3}, t\right)$ is solution to the same system (21) (at the first order of $\frac{1}{\lambda}$ ) as $x$ in the observability analysis. Therefore, whenever $\left(\eta\left(x_{3}, t\right), \dot{\eta}\left(x_{3}, t\right)\right)$ is invertible, $\chi\left(x_{3}, t\right)$ corresponds to $x$ in the observability analysis, and further ||$\chi\left(x_{3}, t\right)-\left.L i\right|^{2}-\Phi^{2} \mid$ corresponds to $P\left(x_{3}, t\right)$, still at the first order in $\frac{1}{\lambda}$. Thus, in order to find $x_{3}$, one could minimize ||$\chi\left(x_{3}, t\right)-\left.L i\right|^{2}-\Phi^{2} \mid$ instead of finding the roots of $J$. But the injection of the input $i$ in the criterion increases its sensitivity to noise.

\footnotetext{
${ }^{2}$ The annulus $C\left(a, r_{0}, r_{1}\right)$ is the set of points such that $r_{0}<|x-a|<r_{1}$.
} 


\subsubsection{About Equation (38)}

(38) implies that, for large values of $\lambda_{i}$, the $J\left(x_{3}, t\right)$ can be approximated by $\frac{P\left(x_{3}, t\right)}{\operatorname{det}\left(\eta\left(x_{3}, t\right), \dot{\eta}\left(x_{3}, t\right)\right)^{2}}$ which is also a rational fraction with numerator of degree 6 and denominator of degree 4 . Therefore, we can hope that, by choosing $\lambda_{i}$ sufficiently large, one can ensure that $J$ does not have more roots than $P$, and the roots of $J$ are close to those of $P$. Since $P$ is perfectly known with Corollary 1 when $\omega, i_{d}$ and $i_{q}$ are constant, it is possible to state the following result.

Theorem 6. Assume the inputs $(u, i)$ and their derivatives are bounded, and that $\omega, i_{d}$ and $i_{q}$ are constant with $\omega \neq 0$ and $i_{d} \neq 0$. Pick three distinct positive real numbers $\left(\tilde{\lambda}_{1}, \tilde{\lambda}_{2}, \tilde{\lambda}_{3}\right)$ and select the $\lambda_{i}$ in the observer as

$$
\left(\lambda_{1}, \lambda_{2}, \lambda_{3}\right)=\left(\alpha \tilde{\lambda}_{1}, \alpha \tilde{\lambda}_{2}, \alpha \tilde{\lambda}_{3}\right) .
$$

with $\alpha$ a positive real number to be tuned. Then, for any initial conditions in the filters, there exists $\bar{t}_{0}>0$, and for any $0<\varepsilon<1$, there exist $\underline{\alpha}>0$, such that for all $\alpha \geq \underline{\alpha}$, for all $t \geq \bar{t}_{0} \frac{\ln \alpha}{\alpha}$, by defining $\underline{r_{\varepsilon}}$ as in Theorem 5, the function $x_{3} \mapsto J\left(x_{3}, t\right)$ admits in $\left[R-\underline{r_{\varepsilon}}, R+\underline{r_{\varepsilon}}\right]$

- only one zero $\hat{R}_{1}(t)$ if $i_{q}>\frac{1-\varepsilon}{2}|i|$;

- two distinct zeros $\left(\hat{R}_{1}(t), \hat{R}_{2}(t)\right)$ if $0<i_{q}<\frac{1-\varepsilon}{2}|i|$.

- only one zero $\hat{R}_{1}(t)$ if $i_{q}=0$

Proof. The proof of this result relies on Rouché's theorem. See Appendix .5.

Remark 2. Unfortunately, we cannot say anything about the number of zeros of $x_{3} \mapsto J\left(x_{3}, t\right)$ outside of $\left[R-\underline{r_{\varepsilon}}, R+\underline{r_{\varepsilon}}\right]$. Indeed, $x_{3} \mapsto J\left(x_{3}, t\right)$ admits (complex) poles outside of $B_{\underline{r_{\varepsilon}}}(R)$ (the roots of $\left.x_{3} \mapsto \operatorname{det}\left(\mathcal{M}\left(x_{3}, t\right)\right)\right)$, and Rouché's theorem would only tell us that it admits at most 6 zeros, which we already know.

We conclude from this study that when $|\omega|$ is lower-bounded away from zero, the invertibility of $\mathcal{M}(R, t)$ (and lower-boundedness of $|\operatorname{det}(\mathcal{M}(R, t))|$ ) is ensured arbitrarily fast by taking the $\lambda_{i}$ sufficiently large. According to Theorem 4, this means that $\lim _{t \rightarrow+\infty} J(R, t)=0$, so that $R$ appears among the roots of $J$ after a certain time.

In particular, when $\omega, i_{d}$ and $i_{q}$ are constant with $\omega \neq 0$ and $i_{d} \neq 0, J$ has only one or two zeros in the vicinity of $R$. Note that $(\Psi, R)$ and $\left(\Psi_{\delta}, R_{\delta}\right)$ identified in Corollary 1 are both solution to the dynamics. Therefore, Theorem 4 apply to both and we have in fact:

$$
\lim _{t \rightarrow+\infty} J(R, t)=\lim _{t \rightarrow+\infty} J\left(R_{\delta}, t\right)=0
$$

This means that the two zeros of $J$ expected with Theorem 6 are likely to be $R$ and $R_{\delta}=R+\frac{2 \omega \Phi i_{q}}{|i|^{2}}$ asymptotically. In fact, although we are not able to prove it theoretically at this point, simulations seem to indicate that $P(\cdot, t)$ has always only two roots, as soon as $i_{d}(t) \neq 0$ and $\omega(t) \neq 0$. Therefore, $J(\cdot, t)$ has, at least after a certain time, also two roots, with one converging to $R$. 


\section{Estimation algorithm}

Following the results of the previous section, our algorithm consists of two parts :

- a dynamic component made of the filters (30) implemented for three distinct sufficiently large positive real numbers $\lambda_{1}, \lambda_{2}, \lambda_{3}$ and from any initial condition at $t=0$,

- a static component which solves (35) at each time $t$, namely

1. extracts the roots of $x_{3} \mapsto J\left(x_{3}, t\right)$ with $J$ defined in (34c), giving the possible estimates $\hat{R}(t)$ of $R$

2. computes $\chi(\hat{R}(t), t)$ for each candidate $\hat{R}(t)$, with $\chi$ defined in $(34 \mathrm{~b})$, to obtain the possible estimates $\hat{\Psi}(t)$ of $\Psi$, and $\Theta(\hat{\Psi}(t))$ of $\theta$, with $\Theta$ defined in (9).

3. selects the most probable estimate based on the sign of $i_{q}$ or on any other a priori known knowledge.

This algorithm can be multi-valued since it returns the at most 6 possible values of the state. What is crucial to remark is that the dynamic part of the observer is completely independent from the static part. This means that any errors committed in the latter have no impact on the former and a good estimate can always be recovered at the next try.

From a numerical point of view, only the root search/selection in the static part of the algorithm is delicate and we dedicate the rest of this section to its practical implementation. Since $x_{3}$ is onedimensional and we often have a fairly good idea of the interval in which $R$ lies, this can be managed with a one-dimensional grid, which can either be fixed around the initial guess $\hat{R}(0)$ or placed at each iteration around the previously found value $\hat{R}(t)$. This latter option enables to follow the slow variations of $R$ with the temperature. Also, unlike $\hat{\Psi}$ (and thus $\hat{\theta}$ ) which must be computed at every time, it may not be necessary to update $\hat{R}$ at each iteration since $R$ is fairly constant: it is enough to search for the roots of $J(\cdot, t)$ only every $d t_{R}>0$.

Also, note that, as seen in Theorem 5, depending on the initial conditions of the filters, a certain time has to elapse before $\mathcal{M}(R, t)$ becomes invertible and thus $J(R, t)$ defined. A solution is to launch the dynamic part at $t=0$, and wait for a time $\bar{t}$ after which the filters have reached their steady state and "forgotten" their initial condition to start the static part. This happens exponentially fast, depending on the chosen eigenvalues in the filters.

Remark 3. The dynamic part of our estimation strategy necessitates the implementation of 7 filters ( $b_{\lambda}$ and $c_{\lambda}$ of dimension 2, and $a_{\lambda}, d_{\lambda}$ and $e_{\lambda}$ of dimension 1) for three values of $\lambda$, namely 21 filters. An alternative solution with only 14 filters exists, but with a criterion $J$ which explicitly depends on the input $i$. Therefore, in presence of noise, the invertibility of $\chi$ and the estimation of $\hat{R}$ and $\hat{\Psi}$ may be deteriorated, and a compromise between computational burden and robustness to noise must be found. 


\subsection{Static part based on root search on fixed grid}

Assume that the resistance is known to belong and remain in an interval $\left[R_{m}, R_{M}\right]$ of $\mathbb{R}_{\geq 0}$. Choose a positive real number $d t_{R}$ and a grid $\mathcal{G}$ of the interval $\left[R_{m}, R_{M}\right]$. We assume to know the mode of use of the machine, namely the sign $s \in\{1,-1\}$ of $i_{q}$. The static algorithm can be built as followed.

- At each time $t \geq \bar{t}$, computation of

$$
\hat{\Psi}(t)=\chi(\hat{R}, t) \quad, \quad \hat{\theta}(t)=\Theta(\hat{\Psi}(t))
$$

given the current value of $\hat{R}$, with $\Theta$ defined in (9).

- Every $d t_{R}>0$, browse the grid $\mathcal{G}$ in search of the roots of $J\left(x_{3}, t\right)$. When a root $\hat{R}_{i}$ is detected, compute

$$
\begin{aligned}
& \hat{\Psi}_{i}=\chi\left(\hat{R}_{i}, t\right) \\
& \hat{\theta}_{i}=\Theta\left(\hat{\Psi}_{i}\right) \\
& i_{q, i}=I_{q}\left(\hat{\theta}_{i}\right)
\end{aligned}
$$

If $i_{q, i}$ is of the right sign, i.e. $i_{q, i} s \geq 0$, take $\hat{R}=\hat{R}_{i}$. Otherwise, carry on to next root. If no root is found take $\hat{R}=\operatorname{Argmin}_{x_{3} \in \mathcal{G}}\left|J\left(x_{3}, t\right)\right|$.

The root detection can be carried out by detecting a change of sign of $J$. This algorithm is based on the fact that $R \in\left[R_{m}, R_{M}\right]$ and $\lim _{t \rightarrow+\infty} J(R, t)=0$ according to the previous section. It also assumes that $R$ is the only root giving $i_{q}$ of the right sign. This is guaranteed by Theorem 3 when $\omega$ and $i_{d q}$ are constant, and this has always been verified in simulations when they are not. Actually, even if two roots with $i_{q}$ of same sign appear, they will be close to one another by continuity, if $\omega$ and $i_{d q}$ do not vary too fast, so that the error made by choosing the wrong one is small. The algorithm can also easily be changed to return all the possible values of $R$, or use extra knowledge to discriminate them.

A drawback of this algorithm is that the grid is constant and may need to be wide if little is known on the evolution of $R$. Achieving the required precision on $R$ may thus necessitate to take a grid of very large dimension, with a heavy computational cost. In the following section, we propose a solution with a possibly smaller dynamical grid and without root search.

\subsection{Static part based on the estimation of $\hat{\omega}=\dot{\hat{\theta}}$}

In the previous algorithm, we do not take advantage of (28) which gives the expression for the other root, when the wrong root is found. In this section, we propose to use this information to avoid the root search and move the grid when no appropriate root is found. This requires the estimation of $\dot{\hat{\theta}}$. More precisely, choose positive real numbers $G$ and $d t_{R}$ and a grid $\mathcal{G}$ of the interval $[-G, G]$. We still assume the sign $s$ of $i_{q}$ is known. The static algorithm can be built as followed. 
- At each time $t \geq \bar{t}$, computation of

$$
\hat{\Psi}(t)=\chi(\hat{R}, t) \quad, \quad \hat{\theta}(t)=\Theta(\hat{\Psi}(t))
$$

given the current value of $\hat{R}$, with $\Theta$ defined in (9).

- At each time $t$, estimation of $\hat{\omega}(t)=\dot{\hat{\theta}}(t)$ (see below).

- Every $d t_{R}>0$, update of the value of $\hat{R}$ with the following algorithm:

$$
\begin{aligned}
& \hat{R}_{1}=\operatorname{Argmin} \\
& \hat{\Psi}_{1}=\chi\left(\hat{R}_{1}, t\right) \\
& \hat{\theta}_{1}=\Theta\left(\hat{\Psi}_{1}\right) \\
& i_{q, 1}=I_{q}\left(\hat{\theta}_{1}\right) \\
& \text { if } i_{q, 1} \geq\left|J\left(x_{3}, t\right)\right| \\
& \quad \hat{R}=\hat{R}_{1} \\
& \text { else } \\
& \quad \hat{R}_{2}=\hat{R}_{1}+\frac{2 \Phi \hat{\omega}(t) i_{q, 1}}{|i(t)|^{2}} \\
& \quad \delta=\hat{R}_{2}-\hat{R}_{1} \\
& \quad \text { if }|\delta|>G \text { then } \\
& \quad \hat{R}=\hat{R}_{2} \\
& \text { else if } \delta>0 \text { then } \\
& \quad \hat{R}=\operatorname{Argmin}_{x_{3} \in \hat{R}+\left(\mathcal{G} \cap\left[\frac{\delta}{2}, G\right]\right)}\left|J\left(x_{3}, t\right)\right| \\
& \text { else } \\
& \quad \hat{R}=\operatorname{Argmin}_{x_{3} \in \hat{R}+\left(\mathcal{G} \cap\left[-G, \frac{\delta}{2}\right]\right)}\left|J\left(x_{3}, t\right)\right| \\
& \text { end if } \\
& \text { end if }
\end{aligned}
$$

In other words, the minimum of $J$ is computed on the grid $\hat{R}+\mathcal{G}$ centered at the current value $\hat{R}$ and, if the corresponding $i_{q}$ is of right sign, this value is kept. Otherwise, we take the other candidate given by Theorem 3, or rather, if this other value is in the grid where $J$ has already been computed, we take the true minimum of $J$ around that value. This latter option offers the possibility of correcting the estimate given by Theorem 3 when $\omega, i_{d}$ and $i_{q}$ are not constant and/or when $\hat{w}$ is not exact.

In order to implement this algorithm, $\hat{\omega}=\dot{\hat{\theta}}$ needs to be estimated. But observe that it is enough to have an approximate estimation of $\hat{\omega}$ since its value is only used to know in which part of the grid the other root is, or to move the grid to a place where $R$ is more likely to be at the next iteration. An estimator for $\hat{\omega}$ can be designed neglecting its dynamics in numerous ways, including dirty derivatives, exact differentiators etc. See $[25,(18)]$ for example. Here to exploit the 
fact that $\hat{\theta}$ lives in a circle and not in the real line, we design our estimator from the model

$$
\left\{\begin{aligned}
\dot{\chi}_{s} & =-\ell \chi_{s}-\left[\ell^{2}+\hat{\omega}^{2}\right] \sin (\hat{\theta}), \\
\dot{\chi}_{c} & =-\ell \chi_{c}-\left[\ell^{2}+\hat{\omega}^{2}\right] \cos (\hat{\theta}), \\
\dot{\hat{\omega}} & =0
\end{aligned}\right.
$$

obtained from $\dot{\hat{\theta}}=\hat{\omega}, \dot{\hat{\omega}}=0$ and the coordinates

$$
\chi_{c}=-\hat{\omega} \sin (\hat{\theta})-\ell \cos (\hat{\theta}), \quad \chi_{s}=\hat{\omega} \cos (\hat{\theta})-\ell \sin (\hat{\theta}) .
$$

The interest of the model (39) is twofold. One, when $\hat{\omega}$ is known and $(\sin (\hat{\theta}), \cos (\hat{\theta}))$ is an input, an observer for $\left(\chi_{s}, \chi_{c}\right)$ is obtained by simply copying the dynamics and choosing $\ell$ strictly positive. Second, we can obtain $\hat{\omega}$ as

$$
\hat{\omega}^{2}=\chi_{c}^{2}+\chi_{s}^{2}-\ell^{2} \quad \text { or } \quad \hat{\omega}=\chi_{s} \cos (\hat{\theta})-\chi_{c} \sin (\hat{\theta}) .
$$

Then an estimation of $\hat{\omega}$ is given by

$$
\left\{\begin{aligned}
\dot{\hat{\chi}}_{s} & =-\ell \hat{\chi}_{s}-\left[\ell^{2}+\hat{\varpi}\right] \sin (\hat{\theta}) \\
\dot{\hat{\chi}}_{c} & =-\ell \hat{\chi}_{c}-\left[\ell^{2}+\hat{\varpi}\right] \cos (\hat{\theta}) \\
\dot{\hat{\varpi}} & =k\left(\left[\hat{\chi}_{c} \cos (\hat{\theta})+\hat{\chi}_{s} \sin (\hat{\theta})\right]+\ell\right), \\
\hat{\omega}_{e} & =\hat{\chi}_{s} \cos (\hat{\theta})-\hat{\chi}_{c} \sin (\hat{\theta})
\end{aligned}\right.
$$

obtained from (39) and (40) via a very basic Lyapunov design of adaptive observers.

Lemma 3. If $\hat{\omega}=\dot{\hat{\theta}}$ is constant, for any positive scalars $k$ and $\ell$, any solution to (41) verifies $\lim _{t \rightarrow+\infty} \hat{\omega}_{e}-\hat{\omega}=0$. Otherwise, for any positive scalars $\omega_{m}, d \omega_{m}, \varepsilon$ and $k_{0}$, there exists $\ell^{*}>0$ such that for any $\ell \geq \ell^{*}$, any solution to (41) with $k=\ell^{\alpha} k_{0}, \alpha \in(0,1],|\hat{\omega}| \leq \omega_{m}$ and $|\dot{\hat{\omega}}| \leq d \omega_{m}$ verifies $\left|\hat{\omega}_{e}-\hat{\omega}\right| \leq \varepsilon$ after a certain time.

Proof. If $\hat{\omega}$ is constant, the claim is a direct consequence of

$$
\overbrace{\left[\hat{\chi}_{c}-\chi_{c}\right]^{2}+\left[\hat{\chi}_{s}-\chi_{s}\right]^{2}+\frac{1}{k}\left[\hat{\varpi}-\hat{\omega}^{2}\right]^{2}}=-2 \ell\left(\left[\hat{\chi}_{c}-\chi_{c}\right]^{2}+\left[\hat{\chi}_{s}-\chi_{s}\right]^{2}\right)
$$

which ensures that $\lim \hat{\chi}_{c}-\chi_{c}=0, \lim \hat{\chi}_{s}-\chi_{s}=0$, so that $\lim \hat{\omega}_{e}=\hat{\omega}$. This then also implies that $\lim \hat{\omega}=\hat{\omega}^{2}$. Let us now consider the general case where $\hat{\omega}$ is not necessarily constant. Assume though that $\hat{\omega}$ and $\dot{\hat{\omega}}$ are bounded. Letting $e_{\chi_{c}}=\hat{\chi}_{c}-\chi_{c}, e_{\chi_{s}}=\hat{\chi}_{s}-\chi_{s}, e_{\varpi}=\hat{\varpi}-\hat{\omega}^{2}$, we are going to show the practical convergence of $e_{\chi_{c}}$ and $e_{\chi_{s}}$ by taking $k$ and $\ell$ sufficiently large. The practical convergence of $\hat{\omega}_{e}$ will follow. Denoting $e=\left(e_{\chi_{c}}, e_{\chi_{s}}, e_{\varpi}\right)$, we have

$$
\dot{e}=A(t) e+\Delta
$$


with

$$
A(t)=\left(\begin{array}{cc}
-\ell I & -b \\
k b^{\top} & 0
\end{array}\right) \quad, \quad I=\left(\begin{array}{ll}
1 & 0 \\
0 & 1
\end{array}\right) \quad, \quad b=\left(\begin{array}{c}
\cos \hat{\theta} \\
\sin \hat{\theta}
\end{array}\right) \quad, \quad \Delta=\left(\begin{array}{c}
\dot{\hat{\omega}} \sin \hat{\theta} \\
-\dot{\hat{\omega}} \cos \hat{\theta} \\
-2 \hat{\omega} \dot{\hat{\omega}}
\end{array}\right)
$$

with

$$
|b|=1 \quad, \quad|\dot{b}| \leq \omega_{m} .
$$

Consider the two-dimensional vector $v$ solution to

$$
\dot{v}=-\ell v-b-k b^{\top} v v \quad, \quad v(0)=-\frac{b(0)}{\ell}
$$

and the change of variable

$$
z=\left(\begin{array}{c}
e_{\chi_{c}} \\
e_{\chi_{s}}
\end{array}\right)-e_{\varpi} v
$$

The error system is transformed into

$$
\begin{aligned}
\dot{z} & =-\ell z-k v b^{\top} z+\Delta_{z} \\
\dot{e}_{\varpi} & =k b^{\top} v e_{\varpi}+k b^{\top} z+\Delta_{\varpi},
\end{aligned}
$$

namely it is triangularized, with

$$
\left|\Delta_{z}\right| \leq d \omega_{m}+2 \omega_{m} d \omega_{m}|v| \quad, \quad \Delta_{\varpi} \leq 2 \omega_{m} d \omega_{m} .
$$

The first step is to show that $v$ is bounded and more precisely

$$
v=-\frac{b}{\ell}+O\left(\frac{1}{\ell^{2}}\right) \text {. }
$$

So denote

$$
\delta_{v}=v+\frac{b}{\ell}
$$

which verifies

$$
\dot{\delta}_{v}=-\left(\ell-\frac{k}{\ell}\right) \delta_{v}+r_{v} \quad, \quad \delta_{v}(0)=0
$$

with

$$
r_{v}=\frac{k}{\ell} b b^{\top} \delta_{v}-\frac{k}{\ell^{2}} b-k \delta_{v} b^{\top} \delta_{v}+\frac{\dot{b}}{\ell} .
$$

Consider $\mu>0$. Since $\delta_{v}(0)=0$, there exists $T \in \mathbb{R}_{>0} \cup\{+\infty\}$ such that $[0, T)$ is the maximal interval such that $\delta_{v}(t)<\mu$ for all $t \in[0, T)$. Then, for all $t \in[0, T)$,

$$
\left|r_{v}\right| \leq \frac{k}{\ell} \mu+\frac{k}{\ell^{2}}+k \mu^{2}+\frac{\omega_{m}}{\ell}
$$

so that

$$
\left|\delta_{v}(t)\right|<\frac{\frac{k}{\ell} \mu+\frac{k}{\ell^{2}}+k \mu^{2}+\frac{\omega_{m}}{\ell}}{\ell-\frac{k}{\ell}} .
$$


Then, necessarily, $T=+\infty$ if

$$
\frac{\frac{k}{\ell} \mu+\frac{k}{\ell^{2}}+k \mu^{2}+\frac{\omega_{m}}{\ell}}{\ell-\frac{k}{\ell}} \leq \mu
$$

This is verified with

$$
\mu=\frac{\ell-\frac{2 k}{\ell}-\sqrt{\left(\ell-\frac{2 k}{\ell}\right)^{2}-4 k\left(\frac{k}{\ell^{2}}+\frac{\omega_{m}}{\ell}\right)}}{2 k}>0
$$

if

$$
\left(\ell-\frac{2 k}{\ell}\right)^{2}>4 k\left(\frac{k}{\ell^{2}}+\frac{\omega_{m}}{\ell}\right) \text {. }
$$

Using the formula

$$
\frac{a_{1}-a_{2}}{a_{3}}=\frac{a_{1}^{2}-a_{2}^{2}}{a_{3}\left(a_{1}+a_{2}\right)}
$$

we get

$$
\mu=\frac{2\left(\frac{k}{\ell^{2}}+\frac{\omega_{m}}{\ell}\right)}{\left(\ell-\frac{2 k}{\ell}+\sqrt{\left(\ell-\frac{2 k}{\ell}\right)^{2}-4 k\left(\frac{k}{\ell^{2}}+\frac{\omega_{m}}{\ell}\right)}\right)} \leq \frac{2\left(\frac{k}{\ell}+\omega_{m}\right)}{\ell^{2}\left(1-\frac{2 k}{\ell^{2}}\right)},
$$

if (42) holds. So choosing $k=k_{0} \ell^{\alpha}, \alpha \in(0,1]$, there exist $\ell_{0}^{*}, \mu_{0}: \mathbb{R} \rightarrow \mathbb{R}$ and $\mu_{0, m}>0$ such that for all $\ell \geq \ell_{0}^{*}, \mu$ is well-defined and

$$
\delta_{v}(t) \leq \frac{\mu_{0}(t)}{\ell^{2}} \quad, \quad \mu_{0}(t) \leq \mu_{0, m} \quad \forall t
$$

So there exists $\Delta_{m}$ such that for all $\ell \geq \ell_{0}^{*},\left|\Delta_{z}(t)\right| \leq \Delta_{m}$ for all $t$, and the error system can be rewritten as

$$
\begin{aligned}
\dot{z} & =-\ell z+\frac{k_{0}}{\ell^{1-\alpha}} b b^{\top} z-\frac{k_{0}}{\ell^{2-\alpha}} \mu_{0} b^{\top} z+\Delta_{z} \\
\dot{e}_{\varpi} & =-\frac{k_{0}}{\ell^{1-\alpha}} e_{\gamma}+\frac{k_{0}}{\ell^{2-\alpha}} b^{\top} \mu_{0} e_{\varpi}+k_{0} \ell^{\alpha} b^{\top} z+\Delta_{\varpi} .
\end{aligned}
$$

It is straightforward to see that $\tilde{z}=\ell z$ verifies

$$
\frac{1}{\ell} \dot{\tilde{z}}=-\tilde{z}+\frac{k_{0}}{\ell^{2-\alpha}} b b^{\top} \tilde{z}-\frac{k_{0}}{\ell^{3-\alpha}} \mu_{0} b^{\top} \tilde{z}+\Delta_{z}
$$

whose trajectories can be bounded independently from $\ell$ for $\ell$ sufficiently large (with a reasoning similar to the one with $\mu$ above). It follows that

$$
\dot{e}_{\varpi}=-\frac{k_{0}}{\ell^{1-\alpha}} e_{\varpi}+\frac{k_{0}}{\ell^{2-\alpha}} b^{\top} \mu_{0} e_{\varpi}+\frac{k_{0}}{\ell^{1-\alpha}} b^{\top} \tilde{z}+\Delta_{\varpi},
$$

so that $\tilde{e}_{\varpi}=\frac{e_{\varpi}}{\ell^{1-\alpha}}$ verifies

$$
\ell^{1-\alpha} \dot{\tilde{e}}_{\varpi}=-k_{0} \tilde{e}_{\varpi}+\frac{k_{0}}{\ell} b^{\top} \mu_{0} \tilde{e}_{\varpi}+\frac{k_{0}}{\ell^{1-\alpha}} b^{\top} \tilde{z}+\Delta_{\varpi}
$$


whose trajectories can be bounded independently from $\ell$ for $\ell$ sufficiently large in the same way. Finally, we conclude that

$$
\left(\begin{array}{l}
e_{\chi_{c}} \\
e_{\chi_{s}}
\end{array}\right)=\frac{\tilde{z}}{\ell}+\tilde{e}_{\varpi}\left(-\frac{b}{\ell^{\alpha}}+\frac{\mu_{0}}{\ell^{1+\alpha}}\right) \quad \forall t,
$$

with $\tilde{z}, b, \tilde{e}_{\varpi}$ and $\mu_{0}$ bounded independently from $\ell$, we conclude that $e_{\chi_{c}}$ and $e_{\chi_{s}}$ can be made arbitrarily small by taking $k=k_{0} \ell^{\alpha}$ with $\alpha \in(0,1]$ and $\ell$ sufficiently large. The result follows for $\hat{\omega}_{e}$. Note that on the other hand, $e_{\varpi}=\ell^{1-\alpha} \tilde{e}_{\varpi}$ cannot be made small, and even increases with $\ell$.

When $\hat{\omega}$ is not constant, practical convergence is obtained for $\hat{\chi}_{c}$ and $\hat{\chi}_{s}$, but not for $\hat{\varpi}$, hence the interest of $(40)$.

Notice that $\hat{\theta}$ is used to estimate $\hat{\omega}$ which in turn is used in the estimation algorithm of $\theta$. This may appear circular. However, the estimate of $\hat{\omega}$ is only used to move the grid and "switch"

the basin of attraction when the sign of $\hat{i_{q}}$ is not right. This is in particular useful when $R$ is not in the grid and thus enables to take a smaller grid width and have more precision with fewer computations. Actually, to use even less this estimation, we could also combine both algorithms, namely carry out a root search on the grid and use (28) only as a security escape if no root with appropriate sign of $i_{q}$ can be found in the grid. Finally, it is important to note that the filters (30) (and therefore the criterion $J$ ) are completely independent from the static algorithm to recover $\hat{\theta}$ and $\hat{R}$. Any other strategy could be implemented, in particular more efficient ones to avoid full evaluation on the grid (e.g. Newton-Rapson).

\section{Robustness}

\section{$5.1 \quad$ Noise/delays in input signals}

The inputs $u$ and $i$ entering the algorithm are likely to contain noise and phase shifts due to the dynamics of the sensors, as exhibited in [27].

The dynamic part of the algorithm behaves well in presence of noise thanks to the low-pass filters (30) whose eigenvalues can be chosen as part of the design. Regarding the static part, it is important to notice that the criterion $J$ and the map $\chi$ depend only on those filtered versions of the inputs $u$ and $i$. Therefore, the root search and the computation of $\hat{\Psi}$ are little impacted by the noise. Only the computation of $\hat{\theta}$ with (9) and of $\widehat{i_{q}}$ relies on a direct injection of the noisy signal $i$. It follows that the root selection based on the sign of $\widehat{i_{q}}$ could be compromised for small values of $i_{q}$, but (25) suggests that in that case, the two roots are close to each other, thus resulting in a small error on $\hat{R}$. Finally, another source of noise exists in the algorithm of Section 4.2 through the use of $\frac{2 \Phi \hat{\omega}(t) i_{q, 1}}{|i(t)|^{2}}$. However, as pointed out above, this quantity is only used for a rough grid 
positioning or to determine which side of the grid is likely to contain the right root, and a small noise on that quantity have no impact on the estimation.

As for the impact of delays or other disturbances like errors on the parameters $\Phi$ and $L$, a more precise study should be carried out. In this paper, we address those issues only implicitly in Section 6.2 when applying our observer to real data.

\subsection{Modelling errors}

The model on which our observer is based, is only an approximation of reality. For example, $L$ and $\Phi$ are actually not constant, (6) is not valid if the electric signals are not balanced, or if the repartition of the windings and the geometry of the magnet are not perfectly symmetric, namely if the machine is salient. The effects of all these disturbances should be studied, but in this paragraph we concentrate our attention to the effect of saliency. According to [28], this means that (13) is not verified and is replaced by

$$
\Psi=L_{0} i+L_{1}\left(\begin{array}{cc}
\cos 2 \theta & \sin 2 \theta \\
\sin 2 \theta & -\cos 2 \theta
\end{array}\right) i+\Phi\left(\begin{array}{c}
\cos \theta \\
\sin \theta
\end{array}\right)
$$

with $L_{0}$ and $L_{1}$ given by

$$
L_{0}=\frac{L_{d}+L_{q}}{2} \quad, \quad L_{1}=\frac{L_{d}-L_{q}}{2},
$$

where $L_{d}$ and $L_{q}$ are the inductances in the rotating frame. When there is no saliency, $L_{d}=L_{q}$, $L_{1}=0$ and we recover our model (13). But as noticed in [8], (43) is equivalent to

$$
\Psi=\left(L_{0}-L_{1}\right) i+\left(\Phi+2 L_{1} i_{d}(\theta)\right)\left(\begin{array}{c}
\cos \theta \\
\sin \theta
\end{array}\right)
$$

and therefore, when $i_{d}$ is constant, $(\Psi, R)$ is still solution to our model (10) provided we replace $L$ and $\Phi$ by

$$
L_{s}=L_{0}-L_{1} \quad, \quad \Phi_{s}=\left|\Phi+2 L_{1} i_{d}\right|
$$

namely

$$
\left|\Psi-L_{s} i\right|^{2}-\Phi_{s}^{2}=0
$$

Therefore, we could adapt our algorithm replacing $L$ by $L_{s}$ and $\Phi$ by $\Phi_{s}$. The problem is that $\Phi_{s}$ is unknown because $i_{d}$ depends on the unknown $\theta$. However, it was observed in [8] that for any $\Psi$, there exists a unique $\theta=\Theta_{s}(\Psi)$ verifying (44) which can be computed without the knowledge of $\Phi_{s} \mathrm{by}^{3}$ :

$$
\Theta_{s}(\Psi)=\left\{\begin{array}{l}
\arg \left(\Psi-L_{s} i\right), \text { if } \Phi-2 L_{1} I_{d}\left(\arg \left(\Psi-L_{s} i\right)\right)>0 \\
\arg \left(\Psi-L_{s} i\right)+\pi, \text { otherwise }
\end{array}\right.
$$

\footnotetext{
${ }^{3}$ The sign of $\Phi+2 L_{1} i_{d}$ is unknown, so $\theta=\arg \left(\Psi-L_{s} i\right)$ is no longer true.
} 
We can thus consider the following modified function

$$
\tilde{T}\left(x, x_{3}, t\right)=T\left(x, x_{3}, t\right)+m_{\lambda}\left(\Phi^{2}-\Phi_{s}(x)^{2}\right)
$$

where

$$
\Phi_{s}(x)=\left|\Phi+2 L_{1} I_{d}\left(\Theta_{s}(x)\right)\right| .
$$

We can check that, if $\Psi$ verifies (45) instead of (13) with $\Phi_{s}$ (i.e. $i_{d}$ ) constant, $t \rightarrow \tilde{T}(\Psi(t), R, t)$ still exponentially decays to zero when taking $L_{s}$ instead of $L$ in (30) . Now, because $M_{\lambda} m_{\lambda}=0$, $M_{\lambda} \tilde{T}=M_{\lambda} T$ and Theorem 4 still holds with the same function $\chi$ defined in (34b) and criterion

$$
\tilde{J}\left(x_{3}, t\right)=m_{\lambda}^{\top}\left(e(t)-\tilde{T}\left(\chi\left(x_{3}, t\right), x_{3}, t\right)\right)
$$

instead of (34c). Therefore, we can implement the algorithm presented in Section 4.1 but replacing

- $L$ by $L_{s}$ everywhere

- criterion $|J|$ by $|\tilde{J}|$

- the function $\Theta$ by $\Theta_{s}$.

Of course, in presence of saliency, the convergence is ensured only for solutions $(\Psi, R)$ giving a constant (or slowly varying) $i_{d}$. This is illustrated in Section 6.2 on real data.

\section{Simulations}

\subsection{Ideal data with non-salient model}

We start by testing our observer on ideal data produced by a PMSM model of the type (3), under a plausible torque scenario and where the input $u$ is chosen to follow the rotation speed scenario $\omega_{R}$ shown on Figure 1. The corresponding voltages and currents are plotted in Figure 2 in the rotating frame. Note that at $t=3$, although the speed setpoint is constant, an external torque is added, resulting in a transient behavior in the signals. This torque then remains constant throughout the simulation.

The results of the simulations are presented in Figure 3, for two resistance grids with amplitude $G=1$ and $G=0.1$ respectively. At the beginning, we wait for 0.5 s before starting the estimation, to let the filters reach their steady state. The estimation of $\hat{\omega}$ (and thus $\omega$ ) uses observer (41) with $\ell=1000$ and $k=500$ and is shown in Figure 1 .

Observe that with $G=1$, the algorithm finds the right value of $R$ in two iterations only, whereas with $G=0.1$, it takes a longer time before $R$ can appear in the grid. In fact, for a same precision, the broader the grid, the higher the chances of $R$ appearing in it, but the larger the number of points and computation time, and also the higher the chances of having several minima in the grid. In practice, one know roughly well the initial value of the resistance, so that a grid with small 


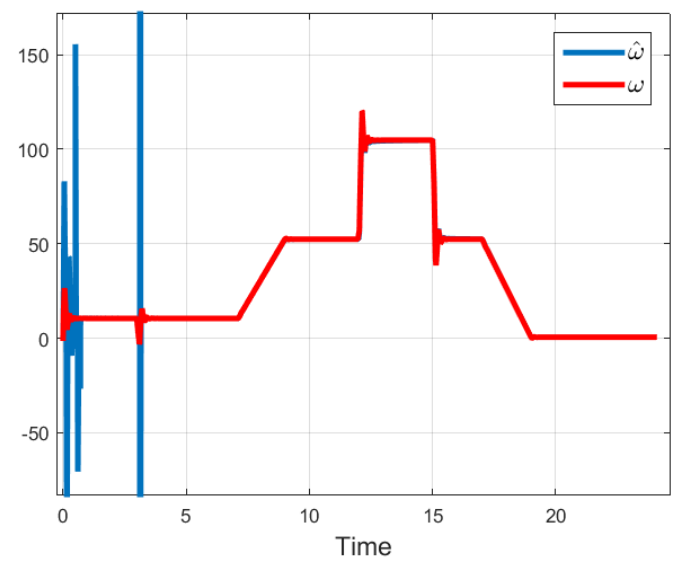

Figure 1: Ideal data: Rotation speed $\omega=\dot{\theta}$ and estimated rotation speed $\hat{\omega}=\dot{\hat{\theta}}$. The estimation algorithm starts at $\bar{t}=0.5$.

amplitude around the initial guess can be chosen, which then follows $R$ throughout the experiment (if it evolves due to temperature for instance).

The evolution of the criterion $J$ during the simulation with $G=1$ is shown in Figure 4. One can see that the minimum is well marked around $R=1.45 \Omega$.

As for the estimation of $\theta$, it naturally converges once $\hat{R}$ has converged. It is interesting to observe the peak in the error around $t=3$ (which in turn appears on $\hat{\omega}$ ) shown on Figure 1 ). This is due to the sudden addition of a torque which destabilizes $i_{d}$ and $\omega$ and makes them go through 0 . We have seen that in this case, observability is lost and $\mathcal{M}(R, t)$ is likely to be non invertible (the assumption of Theorem 5 is no longer verified). This event is not visible on $\hat{R}$ because it is not updated at those precise moments. A very interesting feature here is that this anomaly is not propagated in time. This is because it does not appear in the dynamics of the observer but only in its (static) output function transforming the observer state $\left(a_{\lambda_{i}}, b_{\lambda_{i}}, c_{\lambda_{i}}, d_{\lambda_{i}}, e_{\lambda_{i}}\right)$ in the estimate $\hat{R}_{1}$ and $\hat{\theta}_{1}$.

\subsection{Real data}

In order to test the robustness of our algorithm with respect to noise and saliency, we apply it in open-loop (and offline) to real data obtained from a PMSM used in a test bed at the French institute IFP Energies Nouvelles . For this motor, an identification of the parameters involved in the model (1) with (43) has led to $L_{d}=0.72 \mathrm{mH}, L_{q}=0.78 \mathrm{mH}, \Phi=8.94 \mathrm{mWb}$, and $R=0.151 \Omega$. Note that this motor presents saliency because $L_{d} \neq L_{q}$. The experiment from which the data comes from aimed at keeping the rotation speed constant at a regime of $w_{r}=150 \mathrm{rpm}$ (with $n_{p}=10$, this gives $\omega=157 \mathrm{rad} / \mathrm{s}$ ). We have at our disposal the measurement of the rotor position 


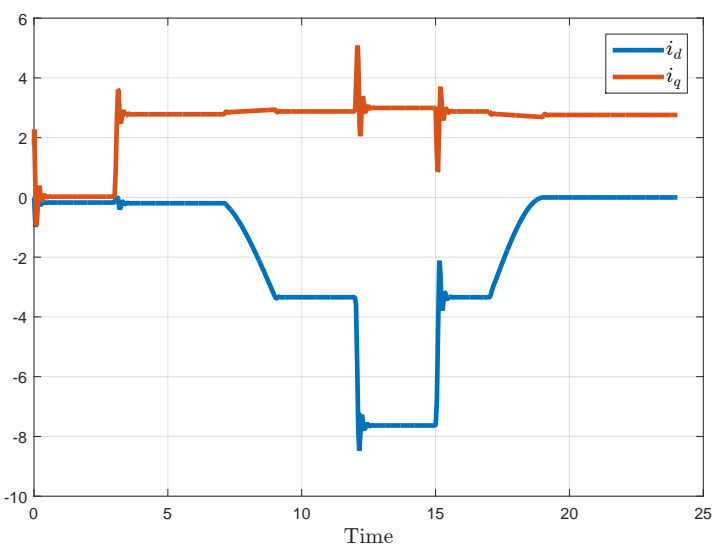

(a) $i_{d q}=\mathcal{R}(-\theta) i$

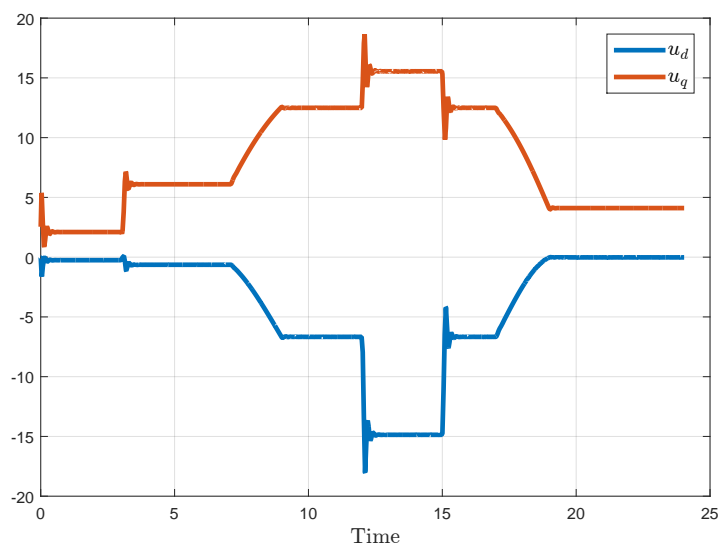

(b) $u_{d q}=\mathcal{R}(-\theta) u$

Figure 2: Ideal data: currents and voltages in the rotating frame.

$\theta_{m}$ for verification and transformations between rotating and fixed frame: it is not used in the algorithm. The recorded measurements of voltages and currents are plotted in Figure 5 in the rotating frame.

The results of a simulation of the observer using the modifications discussed in Section 5.2 to take saliency into account, are presented in Figure 7. The observer manages to reconstruct $\hat{R}$ rapidly and then $\hat{\theta}-\theta$ converges to 0 , with some oscillations due to the noise. The amplitude of these oscillations depends on the chosen eigenvalues $\lambda_{i}$, and a more thorough analysis of this phenomenon should be carried out in order to select those parameters optimally.

Finally, the evolution of the criterion $|\tilde{J}|$ during the simulation is shown in Figure 6 . Because $\omega, i_{d}$ and $i_{q}$ are constant, the criterion $|\tilde{J}(\cdot, t)|$ tends to a constant criterion which has two roots given by $R$ and $R_{\delta}=R+\frac{2 \Phi_{s} \omega i_{q}}{|i|^{2}}$ as predicted by Theorems 3 and 6 .

\section{Conclusion}

Unlike the pair $(\Psi, \Phi)$, the pair $(\Psi, R)$ is not observable for the electric equations $(6)$ of the PMSM model. However, we have proved that when $\omega$ and $i_{d}$ are not constantly zero, only a finite number of indistinguishable trajectories may exist, and they are reduced to two when $\omega, i_{d}$ and $i_{q}$ are constant. Such a property is very different from what we would get if the system was linear and could motivate theoretical research on the fact that this finiteness is generic or rare. Also, our estimation strategy is inspired from the well-established nonlinear Luenberger methodology and enables to recover the maximum 6 indistinguishable candidates. This is interesting because there is currently no theoretical result saying that the inversion of the transformation attached to the Luenberger design enables to recover the indistinguishable trajectories in the absence of 


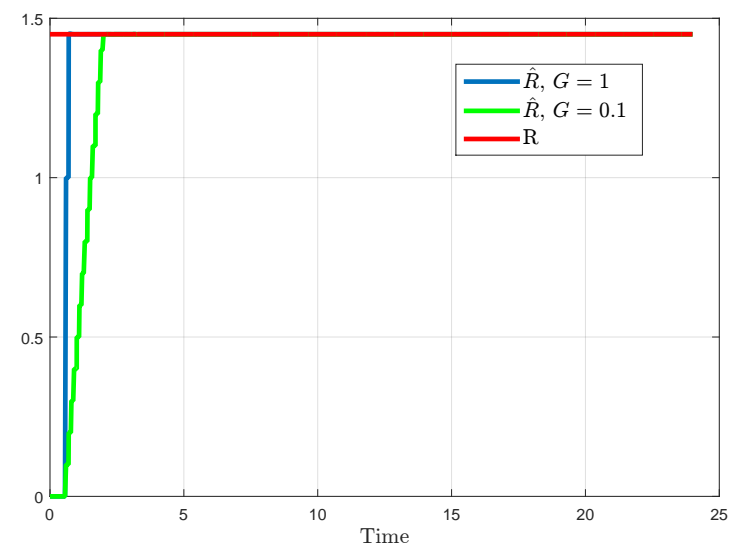

(a) $\hat{R}$

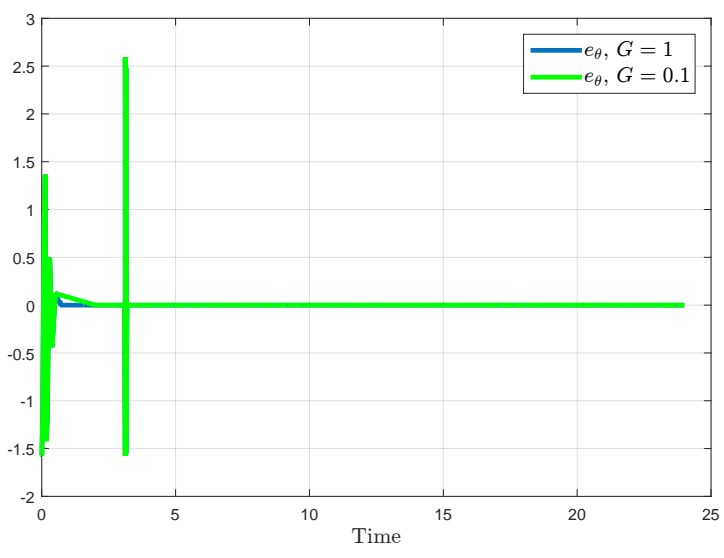

(b) $e_{\theta}=\hat{\theta}-\theta[\mathrm{rad}]$

Figure 3: Ideal data: Results of the observer algorithm discussed in Section 4.1 with $\lambda_{1}=20$, $\lambda_{2}=30, \lambda_{3}=40, d t_{R}=0.1$, and two grids with amplitude $G=1$ and $G=0.1$ respectively. The estimation starts at $\bar{t}=0.5$.
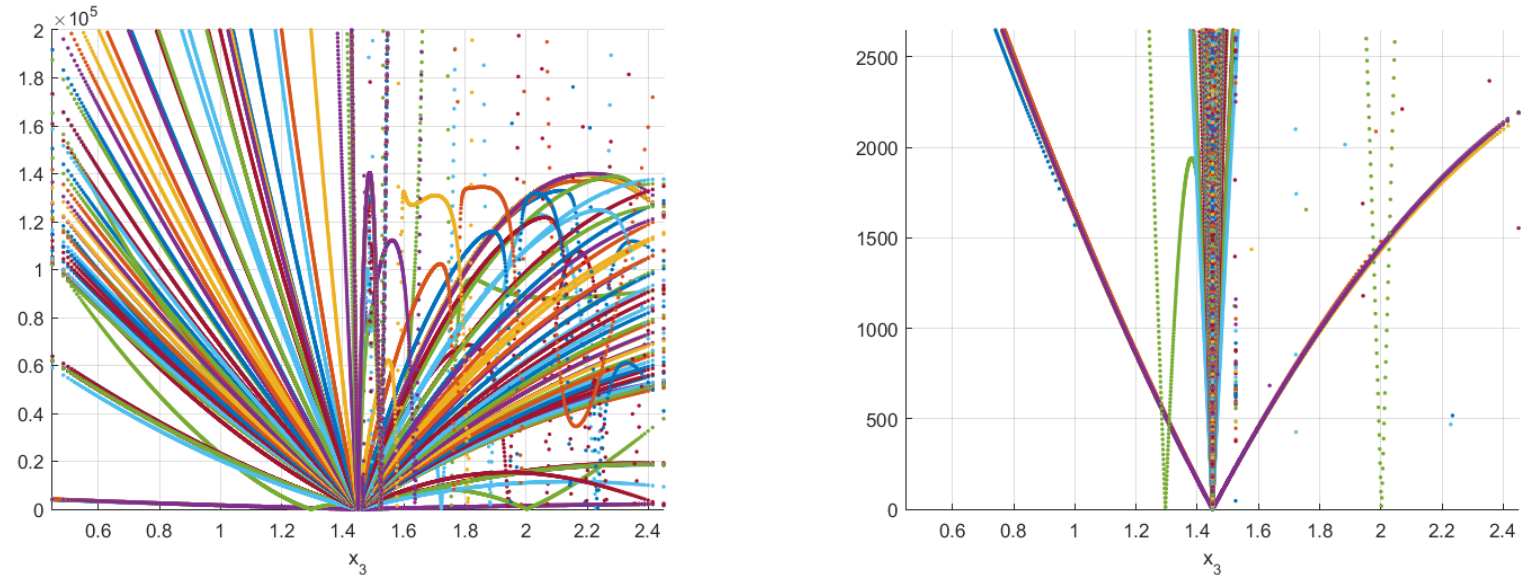

(b) Zoom around $R=1.45$

Figure 4: Ideal data: Plot of the criterion $|J(\cdot, t)|$ on the grid with $G=1$ at each iteration where $\hat{R}$ is updated, i.e. every $d t_{R}=0.1$. 


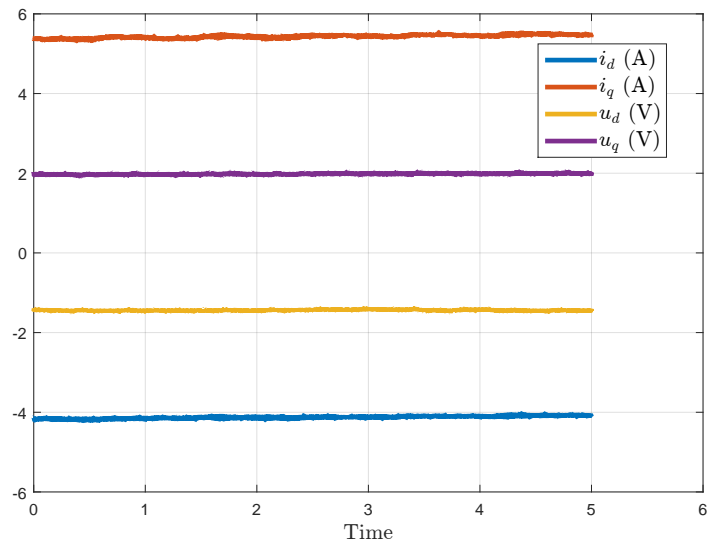

Figure 5: Real data: currents and voltages in the rotating frame.

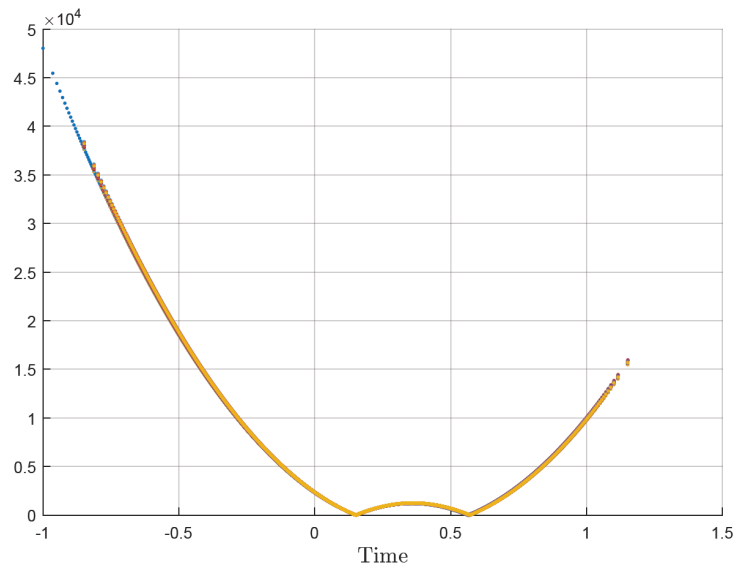

Figure 6: Real data: Plot of the criterion $|J(\cdot, t)|$ on the grid with $G=1$ at each iteration where $\hat{R}$ is updated, i.e. every $d t_{R}=0.1$. 


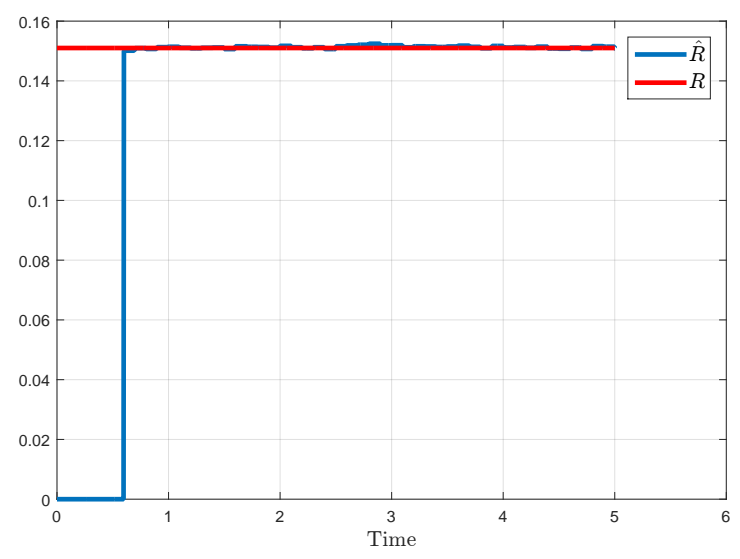

(a) $\hat{R}$

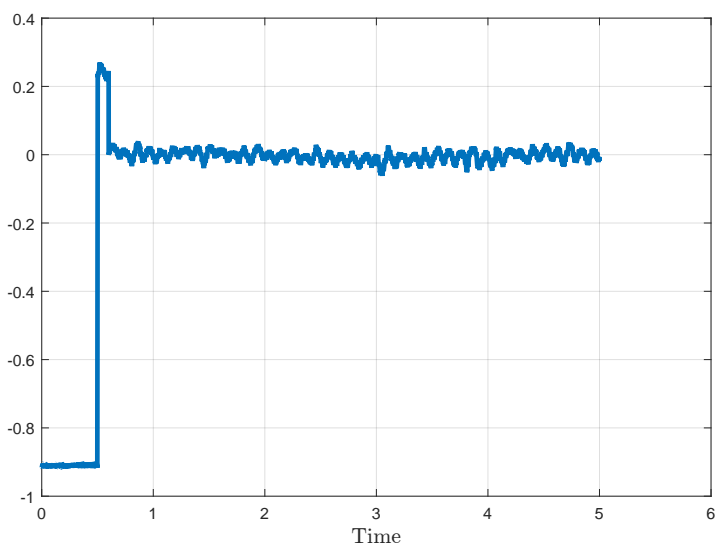

(b) $e_{\theta}=\hat{\theta}-\theta[\mathrm{rad}]$

Figure 7: Real data: Results of the observer algorithm discussed in Section 5.2, with $\lambda_{1}=40$, $\lambda_{2}=50, \lambda_{3}=60, d t_{R}=0.1$, and a grid with amplitude $G=1$. The estimation starts at $t=0.5$.

observability. This may motivate future research on the generality of this peculiar property.

From a more practical point of view, the important robustness to saliency requires in our design either the saliency to be small or that $i_{d}$ be constant/slowly-varying. A way to avoid those assumptions and obtain a more general design would be to carry out the same observability analysis but directly on the salient model (43). Note that simulations of our observer with a salient model in the case where $i_{d}$ is not constant indicate that the criterion obtained in this paper still has a finite number of roots, with $R$ among them. But we do not know how to distinguish them yet.

As for the impact of the noise, the choice of the eigenvalues $\lambda_{i}$ plays an important role and a detailed analysis could help to determine how to choose those parameters optimally. More generally, the impact of unmodelled effects such as delays in $u$ and $i$ needs to be more precisely investigated.

\section{$.1 \quad$ Proof of Theorem 3}

Consider a solution to System (10). According to Corollary 1 , since $y(t)=\dot{y}(t)=\ddot{y}(t)=0$, it is necessarily $(\Psi, R)$ or $\left(\Psi_{\delta}, R_{\delta}\right)$ where, according to $(22), \Psi_{\delta}$ is given by (27). It remains to show that $\left(\Psi_{\delta}, R_{\delta}\right)$ is a solution to System $(10)$.

Using (20), we get

$$
\left|\eta\left(x_{3}, t\right)\right|^{2}=\omega^{2} \Phi^{2}+2\left(R-x_{3}\right) \Phi \omega i_{q}+\left(R-x_{3}\right)^{2}|i|^{2}
$$

and thus, $\left|\eta\left(R_{\delta}, t\right)\right|=\omega \Phi$. It follows that there exists $\theta_{\delta}$ such that

$$
\eta\left(R_{\delta}, t\right)=\omega \Phi\left(\begin{array}{c}
-\sin \theta_{\delta} \\
\cos \theta_{\delta}
\end{array}\right)=-\omega \Phi J z_{\delta}
$$


where we denote $z_{\delta}=\left(\begin{array}{c}\cos \theta_{\delta} \\ \sin \theta_{\delta}\end{array}\right)$. We deduce according to $(20)$ that

$$
-\omega \Phi J z_{\delta}=-\omega \Phi J z+\left(R-R_{\delta}\right) i=-\omega \Phi J z-\frac{2 \Phi \omega i_{q}}{|i|^{2}} i
$$

and after a rotation of angle $-\theta$, we have

$$
J\left(\begin{array}{c}
\cos \left(\theta_{\delta}-\theta\right) \\
\sin \left(\theta_{\delta}-\theta\right)
\end{array}\right)=\left(\begin{array}{c}
0 \\
-1
\end{array}\right)+\frac{2 i_{q}}{|i|^{2}} i_{d q}
$$

Therefore, $\theta_{\delta}-\theta$ is a constant and

$$
w_{\delta}=\dot{\theta_{\delta}}=w .
$$

It follows that $x_{\delta}$ defined by

$$
x_{\delta}=L i+\Phi z_{\delta}
$$

verifies the dynamics:

$$
\dot{x_{\delta}}=L \dot{\hat{i}}-\Phi \omega_{\delta} J z_{\delta}=L \dot{\overparen{i}}-\Phi \omega J z_{\delta}=L \dot{\overparen{i}}+\eta\left(R_{\delta}, t\right)=u-R_{\delta} i
$$

and $0=y=\left|x_{\delta}-L i\right|^{2}-\Phi$. Thus, $\left(x_{\delta}, R_{\delta}\right)$ is a solution to (10), which must appear among $\left\{(\Psi, R),\left(\Psi_{\delta}, R_{\delta}\right)\right\}$, i.e. it is necessarily $\left(\Psi_{\delta}, R_{\delta}\right)$. Therefore, $(\Psi, R)$ and $\left(\Psi_{\delta}, R_{\delta}\right)$ are the only two indistinguishable solutions.

Now, according to (48),

$$
\cos \left(\theta_{\delta}-\theta\right)=1-2 \frac{i_{q}^{2}}{|i|^{2}} \quad, \quad \sin \left(\theta_{\delta}-\theta\right)=2 \frac{i_{q} i_{d}}{|i|^{2}}
$$

But after a rotation of $-\theta_{\delta}$ instead of $\theta$, we would have obtained:

$$
\left(\begin{array}{c}
0 \\
-1
\end{array}\right)=J\left(\begin{array}{c}
\cos \left(\theta-\theta_{\delta}\right) \\
\sin \left(\theta-\theta_{\delta}\right)
\end{array}\right)+\frac{2 i_{q}}{|i|^{2}} i_{d q, \delta}
$$

i.e.

$$
\cos \left(\theta_{\delta}-\theta\right)=1+2 \frac{i_{q} i_{q, \delta}}{|i|^{2}} \quad, \quad \sin \left(\theta_{\delta}-\theta\right)=2 \frac{i_{q} i_{d, \delta}}{|i|^{2}}
$$

which gives the result.

Now, if $\hat{R}=R_{\delta}$, one can find $R$ by computing

$$
R=\hat{R}-\frac{2 \Phi \omega i_{q}}{|i|^{2}}=\hat{R}+\frac{2 \Phi \hat{\omega} \hat{i_{q}}}{|i|^{2}}
$$

and if $\hat{R}=R$, one can find $R_{\delta}$ by computing

$$
R_{\delta}=\hat{R}+\frac{2 \Phi \omega i_{q}}{|i|^{2}}=\hat{R}+\frac{2 \Phi \hat{\omega} \hat{i_{q}}}{|i|^{2}}
$$


Similarly, if $\hat{\theta}=\theta_{\delta}$, then $\theta=\hat{\theta}+\Delta$ with $\Delta$ defined by

$$
\begin{aligned}
& \cos (\Delta)=1-2 \frac{i_{q}^{2}}{|i|^{2}}=1-2 \frac{{\hat{i_{q}}}^{2}}{|i|^{2}} \\
& \sin (\Delta)=-2 \frac{\hat{i}_{q} i_{d}}{|i|^{2}}=2 \frac{\overbrace{i_{q} i_{d}}}{|i|^{2}}
\end{aligned}
$$

and if $\hat{\theta}=\theta$, then $\theta_{\delta}=\hat{\theta}-\Delta$ with $-\Delta$ defined by

$$
\begin{aligned}
& \cos (-\Delta)=1-2 \frac{i_{q}^{2}}{|i|^{2}}=1-2 \frac{{\hat{i_{q}}}^{2}}{|i|^{2}} \\
& \sin (-\Delta)=2 \frac{\hat{i}_{q} i_{d}}{|i|^{2}}=2 \frac{\hat{i}_{q}}{|i|^{2}}
\end{aligned}
$$

\section{.2 Proof of Theorem 4}

Observe that

$$
M_{\lambda} T\left(x, x_{3}, t\right)=M_{\lambda} \Lambda\left(c(t)+x_{3} b(t)\right) x+M_{\lambda} a(t) x_{3}+M_{\lambda} d(t) x_{3}^{2}-M_{\lambda} e(t)
$$

is linear in $x$. This means that for any $x_{3}$ and any $t$ such that the matrix $\mathcal{M}\left(x_{3}, t\right)$ is invertible, $x$ is solution of:

$$
x=\mathcal{M}\left(x_{3}, t\right)^{-1} M_{\lambda}\left(T\left(x, x_{3}, t\right)-M_{\lambda} a(t) x_{3}-M_{\lambda} d(t) x_{3}^{2}+e(t)\right) .
$$

Thus, $\left(x, x_{3}\right)=(\Psi(t), R)$ satisfies this equation for all $t \geq \bar{t}$ and we have

$$
|\Psi(t)-\chi(R, t)| \leq\left|\mathcal{M}(R, t)^{-1}\right|\left|M_{\lambda}\right||T(\Psi(t), R, t)| .
$$

Lemma 1 gives the result if

$$
\left|\mathcal{M}(R, t)^{-1}\right|=\frac{1}{|\operatorname{det}(\mathcal{M}(R, t))|}\left|\mathcal{M}^{*}(R, t)\right|
$$

is upper-bounded in time, where $\mathcal{M}^{*}(R, t)$ is the comatrix of $\mathcal{M}(R, t) . t \mapsto \mathcal{M}^{*}(R, t)$ is a continuous function of the coefficients of $c$ and $b$ which are filtered versions of the bounded input $(u, i)$ and which are thus bounded. Since $|\operatorname{det}(\mathcal{M}(R, t))|$ is lower-bounded away from 0 , the conclusion follows.

\section{.3 Proof of Lemma 2}

It is straightforward to check that the quantity

$$
\mu_{\lambda}\left(x_{3}, t\right)=-\left(c_{\lambda}+x_{3} b_{\lambda}+2 \lambda L i\right)
$$

verifies

$$
\overparen{\cdot} \overbrace{\mu_{\lambda}\left(x_{3}, t\right)}=-\lambda\left(\mu_{\lambda}\left(x_{3}, t\right)-2 \eta\left(x_{3}, t\right)\right)
$$


with $\eta$ defined in (18). This means that $\mu_{\lambda}$ is a filtered version of $2 \eta$. Besides, denoting $\mu=$ $\left(\mu_{\lambda_{1}}, \mu_{\lambda_{2}}, \mu_{\lambda_{3}}\right)^{\top}$ we have

$$
\Lambda \mu\left(x_{3}, t\right)=-\left(\Lambda c+x_{3} \Lambda b+2 L m_{\lambda} i^{\top}\right)
$$

and thus since $M_{\lambda} m_{\lambda}=0$,

$$
\mathcal{M}\left(x_{3}, t\right)=-M_{\lambda} \Lambda \mu\left(x_{3}, t\right) .
$$

Let us further investigate the link between $\mu$ and $\eta$. According to the dynamics (50), $\mu_{\lambda}$ can be developed in the following way:

$$
\mu_{\lambda}\left(x_{3}, t\right)=2 \eta\left(x_{3}, t\right)-\frac{2 \dot{\eta}\left(x_{3}, t\right)}{\lambda}+\frac{2 r_{\mu, \lambda}\left(x_{3}, t\right)}{\lambda^{2}}
$$

where the remainder $r_{\mu, \lambda}$ follows the dynamics:

$$
\overbrace{r_{\mu, \lambda}\left(x_{3}, t\right)}^{\cdot}=-\lambda\left(r_{\mu, \lambda}\left(x_{3}, t\right)-\ddot{\eta}\left(x_{3}, t\right)\right)
$$

This yields

$$
\left|r_{\mu, \lambda}\left(x_{3}, t\right)\right| \leq \exp (-\lambda t)\left|r_{\mu, \lambda}\left(x_{3}, 0\right)\right|+\sup _{s \in[0, t]}\left|\ddot{\eta}\left(x_{3}, s\right)\right|
$$

Since $\ddot{\eta}$ is a polynomial in $x_{3}$ with coefficients depending on the bounded signals $\left(\ddot{u}, \ddot{i}, i^{(3)}\right)$, we obtain

$$
\mu_{\lambda}\left(x_{3}, t\right)=2 \eta\left(x_{3}, t\right)-\frac{2 \dot{\eta}\left(x_{3}, t\right)}{\lambda}+O\left(\frac{1}{\lambda^{2}}\right)
$$

It follows that $\mu_{\lambda}$ can be approximated by $2 \eta-\frac{2 \dot{\eta}}{\lambda}$ for large values of $\lambda$. Therefore,

$$
\begin{aligned}
\mathcal{M} & =-2 M_{\lambda} \Lambda\left(\begin{array}{c}
\eta-\frac{\dot{\eta}}{\lambda_{1}}+O\left(\frac{1}{\lambda_{1}^{2}}\right) \\
\eta-\frac{\dot{\eta}}{\lambda_{2}}+O\left(\frac{1}{\lambda_{2}^{2}}\right) \\
\eta-\frac{\dot{\eta}}{\lambda_{3}}+O\left(\frac{1}{\lambda_{3}^{2}}\right)
\end{array}\right) \\
& =-2\left(\begin{array}{cc}
\lambda_{1} \lambda_{2}\left(\lambda_{2}-\lambda_{1}\right) & \lambda_{1}^{2}-\lambda_{2}^{2} \\
\lambda_{2} \lambda_{3}\left(\lambda_{3}-\lambda_{2}\right) & \lambda_{2}^{2}-\lambda_{3}^{2}
\end{array}\right)\left(\begin{array}{c}
\eta^{\top} \\
\dot{\eta}^{\top}
\end{array}\right)+O(\lambda),
\end{aligned}
$$

and straightforward computations give

$$
\operatorname{det}\left(\mathcal{M}\left(x_{3}, t\right)\right)=4 \lambda_{2}^{2}\left(\lambda_{1}-\lambda_{2}\right)\left(\lambda_{2}-\lambda_{3}\right)\left(\lambda_{3}-\lambda_{1}\right) \operatorname{det}\left(\eta\left(x_{3}, t\right), \dot{\eta}\left(x_{3}, t\right)\right)+O\left(\lambda^{4}\right)
$$

i.e. (36).

Let us now develop $\chi\left(x_{3}, t\right)$ with respect to $\lambda$. To do that, we define $\rho_{\lambda}$ with

$$
\rho_{\lambda}\left(x_{3}, t\right)=-e_{\lambda}+a_{\lambda} x_{3}+d_{\lambda} x_{3}^{2}-\lambda L \mu_{\lambda}^{\top} i-\lambda^{2} L^{2}|i|^{2}+\lambda^{2} \Phi^{2},
$$


which follows the dynamics

$$
\overbrace{\rho_{\lambda}\left(x_{3}, t\right)}^{\cdot}=-\lambda\left(\rho_{\lambda}\left(x_{3}, t\right)-\mu_{\lambda}\left(x_{3}, t\right)^{\top} \eta\left(x_{3}, t\right)\right) .
$$

In other words, by denoting $\left(\rho_{\lambda_{1}}, \rho_{\lambda_{2}}, \rho_{\lambda_{3}}\right)^{\top}$, we obtain

$$
\rho\left(x_{3}, t\right)=-e(t)+a(t) x_{3}+d(t) x_{3}^{2}-L \Lambda \mu i-m_{\lambda} L^{2}|i|^{2}+m_{\lambda} \Phi^{2} .
$$

When $\mathcal{M}$ is invertible, by definition of $\chi$ in $(34 \mathrm{~b})$, we have

$$
\mathcal{M}\left(x_{3}, t\right) \chi\left(x_{3}, t\right)=M_{\lambda}\left(e(t)-a(t) x_{3}-d(t) x_{3}^{2}\right)
$$

and thus since $M_{\lambda} m_{\lambda}=0$ and (51),

$$
\mathcal{M}\left(x_{3}, t\right)\left(\chi\left(x_{3}, t\right)-L i\right)=-M_{\lambda} \rho .
$$

As we did above for $\mu$, it is possible to develop $\rho$ thanks to (57). Indeed, it is straightforward to check that

$$
\rho_{\lambda}\left(x_{3}, t\right)=2\left|\eta\left(x_{3}, t\right)\right|^{2}+\frac{r_{\rho, \lambda}\left(x_{3}, t\right)}{\lambda}
$$

with $\overparen{r_{\rho}\left(x_{3}, t\right)}=-\lambda\left(r_{\rho}\left(x_{3}, t\right)+6 \eta\left(x_{3}, t\right)^{\top} \dot{\eta}\left(x_{3}, t\right)\right)$. In other words

$$
\rho_{\lambda}\left(x_{3}, t\right)=2\left|\eta\left(x_{3}, t\right)\right|^{2}+O\left(\frac{1}{\lambda}\right)
$$

and we have with (55)

$$
\begin{aligned}
& \left(\begin{array}{c}
\eta^{\top} \\
\dot{\eta}^{\top}
\end{array}\right)\left(\chi\left(x_{3}, t\right)-L i\right) \\
& =\frac{1}{2}\left(\begin{array}{ll}
\lambda_{1} \lambda_{2}\left(\lambda_{2}-\lambda_{1}\right) & \lambda_{1}^{2}-\lambda_{2}^{2} \\
\lambda_{2} \lambda_{3}\left(\lambda_{3}-\lambda_{2}\right) & \lambda_{2}^{2}-\lambda_{3}^{2}
\end{array}\right)^{-1} M_{\lambda} \rho+O\left(\frac{1}{\lambda}\right) \\
& =\left(\begin{array}{cc}
\lambda_{1} \lambda_{2}\left(\lambda_{2}-\lambda_{1}\right) & \lambda_{1}^{2}-\lambda_{2}^{2} \\
\lambda_{2} \lambda_{3}\left(\lambda_{3}-\lambda_{2}\right) & \lambda_{2}^{2}-\lambda_{3}^{2}
\end{array}\right)^{-1} M_{\lambda}\left(\begin{array}{l}
|\eta|^{2} \\
|\eta|^{2}
\end{array}\right)+O\left(\frac{1}{\lambda}\right) \\
& =\left(\begin{array}{c}
0 \\
-1
\end{array}\right)|\eta|^{2}+O\left(\frac{1}{\lambda}\right) \text {. }
\end{aligned}
$$

Finally, according to the definitions (31), (49) and (58), it is straightforward to check that

$$
T\left(\chi\left(x_{3}, t\right), x_{3}, t\right)=m_{\lambda}\left(\left|\chi\left(x_{3}, t\right)-L i\right|^{2}-\Phi^{2}\right)-\Lambda \mu\left(x_{3}, t\right)\left(\chi\left(x_{3}, t\right)-L i\right)+\rho\left(x_{3}, t\right) .
$$

It follows that for $x_{3}$ and $t$ making $\left(\eta\left(x_{3}, t\right), \dot{\eta}\left(x_{3}, t\right)\right)$ invertible

$$
\begin{aligned}
J\left(x_{3}, t\right) & =-\left(\lambda_{1}^{4}+\lambda_{2}^{4}+\lambda_{3}^{4}\right)\left(\left|\chi\left(x_{3}, t\right)-L i\right|^{2}-\Phi^{2}\right)+O\left(\lambda^{3}\right) \\
& =\left(\lambda_{1}^{4}+\lambda_{2}^{4}+\lambda_{3}^{4}\right) \frac{P\left(x_{3}, t\right)}{\operatorname{det}\left(\eta\left(x_{3}, t\right), \dot{\eta}\left(x_{3}, t\right)\right)^{2}}+O\left(\lambda^{3}\right) .
\end{aligned}
$$




\section{.4 Proof of Theorem 5}

Assume $\left|\operatorname{det}\left(\eta\left(x_{3}, t\right), \dot{\eta}\left(x_{3}, t\right)\right)\right| \geq \underline{d}$. In order to deduce from (36) that $\left|\operatorname{det}\left(\mathcal{M}\left(x_{3}, t\right)\right)\right| \geq \underline{\delta}$ for all $t$, if $\alpha$ is sufficiently large, we need to bound the term $O\left(\lambda^{4}\right)$ uniformly in time. In the present case (54) is

$$
\left|r_{\mu, \tilde{\lambda}}\left(x_{3}, t\right)\right|=\left|r_{\mu, \tilde{\lambda}}\left(x_{3}, 0\right)\right| e^{-\alpha \tilde{\lambda} t}+\sup _{t \in[0, t]}\left|\ddot{\eta}\left(x_{3}, t\right)\right|
$$

And, since $\ddot{\eta}$ is a polynomial in $x_{3}$ with coefficients depending on the bounded signals $\left(\ddot{u}, \stackrel{\imath}{i}, i^{(3)}\right)$, there exists a polynomial $\mathfrak{R}_{r}$ (time and $\alpha$ independent) such that $\left|r_{\mu, \tilde{\lambda}}\left(x_{3}, t\right)\right| \leq\left|r_{\mu, \tilde{\lambda}}\left(x_{3}, 0\right)\right| e^{-\alpha \tilde{\lambda} t}+$ $\mathfrak{R}_{r}\left(\left|x_{3}\right|\right)$. But (52) gives

$$
r_{\mu, \tilde{\lambda}}\left(x_{3}, 0\right)=\alpha^{2}\left[\frac{\tilde{\lambda}^{2}\left[\mu\left(x_{3}, 0\right)-2 \eta\left(x_{3}, 0\right)\right]}{2}\right]+\alpha \tilde{\lambda} \dot{\eta}\left(x_{3}, 0\right)
$$

So with (49), if $\alpha \geq 1$, there exists a polynomial $\mathfrak{R}_{r, 0}$ depending only on $\tilde{\lambda}$, the initialization of the filters and the input, such that $\left|r_{\mu, \tilde{\lambda}}\left(x_{3}, t\right)\right| \leq \alpha^{2} \mathfrak{R}_{r, 0}\left(\left|x_{3}\right|\right) e^{-\alpha \tilde{\lambda} t}+\mathfrak{R}_{r}\left(\left|x_{3}\right|\right)$. Therefore, there exist $\bar{t}_{0}$ and a polynomial $\bar{\Re}_{r}$, both depending only on $\tilde{\lambda}_{i}$ and the initial conditions in the filters, such that, denoting $\bar{t}(\alpha)=\bar{t}_{0} \frac{\ln \alpha}{\alpha},\left|r_{\mu, \tilde{\lambda}}\left(x_{3}, t\right)\right| \leq \overline{\mathfrak{R}}_{r}\left(\left|x_{3}\right|\right)$ for all $t \geq \bar{t}(\alpha)$. Following this term in (55), and then in its determinant (36), the reader can check that $O\left(\lambda^{4}\right)$ in (36) is also a polynomial in $x_{3}$ with bounded (in time) coefficients, at least after $\bar{t}$. Thus, there exists a polynomial $\mathfrak{R}$ (time and $\alpha$ independent) such that for all $\alpha \geq 1$ and all $t \geq \bar{t}(\alpha)$

$$
\frac{1}{\alpha^{5}}\left|\operatorname{det}\left(\mathcal{M}\left(x_{3}, t\right)\right)\right| \geq 4 \tilde{\lambda}_{2}^{2}\left(\tilde{\lambda}_{1}-\tilde{\lambda}_{2}\right)\left(\tilde{\lambda}_{2}-\tilde{\lambda}_{3}\right)\left(\tilde{\lambda}_{3}-\tilde{\lambda}_{1}\right) \underline{d}-\frac{\mathfrak{R}\left(\left|x_{3}\right|\right)}{\alpha}
$$

which is positive when $\alpha$ is sufficiently large.

If besides $|\omega(t)| \geq \underline{\omega}>0$, according to $(24)$,

$$
|\operatorname{det}(\eta(R, t), \dot{\eta}(R, t))|=\left|\omega^{3} \Phi^{2}\right| \geq \underline{\omega}^{3} \Phi^{2}>0
$$

for all $t$, hence the second point of the result.

Assume now that $\omega, i_{d}$ and $i_{q}$ are constant, with $\omega \neq 0$ and $i_{d} \neq 0$. As seen in the proof of Corollary $1, \operatorname{det}(\eta, \dot{\eta})$ and $P$ are time independent polynomials with

$$
\begin{gathered}
\operatorname{det}(\eta, \dot{\eta})\left(x_{3}\right)=\omega^{3} \Phi^{2}\left(1+\frac{\left(R-x_{3}\right)}{\omega \Phi} 2 i_{q}+\frac{\left(R-x_{3}\right)^{2}}{\omega^{2} \Phi^{2}}|i|^{2}\right) \\
P\left(x_{3}\right)=-\Phi^{2} \operatorname{det}\left(\eta\left(x_{3}\right), \dot{\eta}\left(x_{3}\right)\right)^{2} \frac{\left(R-x_{3}\right)}{\omega \Phi}\left(2 i_{q}+\frac{\left(R-x_{3}\right)}{\omega \Phi}|i|^{2}\right) .
\end{gathered}
$$

Therefore, the roots of $\operatorname{det}\left(\eta\left(x_{3}\right), \dot{\eta}\left(x_{3}\right)\right)$ are the complex numbers $\frac{\Phi \omega}{|i|^{2}}\left(-i_{q} \pm j i_{d}\right)$, both situated on the circle with center $R$ and radius $\frac{\Phi \omega}{|i|}$, and

$$
\begin{aligned}
Q\left(x_{3}\right) & =\frac{P\left(x_{3}\right)}{\operatorname{det}\left(\eta\left(x_{3}\right), \dot{\eta}\left(x_{3}\right)\right)^{2}} \\
& =-\Phi^{2} \frac{\left(R-x_{3}\right)}{\omega \Phi}\left(2 i_{q}+\frac{\left(R-x_{3}\right)}{\omega \Phi}|i|^{2}\right)
\end{aligned}
$$


is a polynomial of degree 2 , with the two roots $\left(R, R_{\delta}\right)=\left(R, R+\frac{2 \omega \Phi i_{q}}{|i|^{2}}\right)$ identified in Corollary 1 .

Now, take any $\varepsilon>0$ and consider $\Gamma_{r_{\varepsilon}}(R)$ and $\Gamma_{\overline{r_{\varepsilon}}}(R)$, the circles with center $R$ and radius $\underline{r_{\varepsilon}}$ and $\overline{r_{\varepsilon}}$ respectively. The polynomial $\underline{\operatorname{det}}\left(\eta\left(x_{3}\right), \dot{\eta}\left(x_{3}\right)\right)$ has no root on those circles so that it can be lower-bounded by some $\underline{d}>0$. Also, $\mathfrak{R}$ introduced in (61) is continuous on those compact sets and is bounded by some $\bar{\Re}$. Choosing $\left(\lambda_{1}, \lambda_{2}, \lambda_{3}\right)$ as suggested in the theorem and denoting $\gamma=4 \tilde{\lambda}_{2}^{2}\left(\tilde{\lambda}_{1}-\tilde{\lambda}_{2}\right)\left(\tilde{\lambda}_{2}-\tilde{\lambda}_{3}\right)\left(\tilde{\lambda}_{3}-\tilde{\lambda}_{1}\right)$, we have for all $t \geq \bar{t}(\alpha)$ and all $x_{3}$ in $\Gamma_{\overline{r_{\varepsilon}}}(R) \cup \Gamma_{\underline{r_{\varepsilon}}}(R)$,

$$
\left|\frac{1}{\alpha^{5}} \operatorname{det}\left(\mathcal{M}\left(x_{3}, t\right)\right)-\gamma \operatorname{det}\left(\eta\left(x_{3}\right), \dot{\eta}\left(x_{3}\right)\right)\right| \leq \frac{\mathfrak{R}\left(\left|x_{3}\right|\right)}{\alpha} \leq \frac{\bar{\Re}}{\alpha} \leq \gamma \underline{d} \leq\left|\gamma \operatorname{det}\left(\eta\left(x_{3}\right), \dot{\eta}\left(x_{3}\right)\right)\right|
$$

for $\alpha$ sufficiently large. Both functions being holomorphic (polynomials), according to Rouché's theorem, $\operatorname{det}\left(\mathcal{M}\left(x_{3}, t\right)\right)$ and $\operatorname{det}\left(\eta\left(x_{3}\right), \dot{\eta}\left(x_{3}\right)\right)$ have the same number of $\operatorname{roots} \operatorname{in}^{4} B_{\underline{r_{\varepsilon}}}(R)$ (resp $\left.B_{\overline{r_{\varepsilon}}}(R)\right)$, namely no roots in $B_{\underline{r_{\varepsilon}}}(R)$ (resp 2 roots in $\left.B_{\overline{r_{\varepsilon}}}(R)\right)$. Since we know $\operatorname{det}\left(\mathcal{M}\left(x_{3}, t\right)\right)$ is a polynomial of degree 2 , we deduce that its only two roots are situated in the annulus $C\left(R, \underline{r_{\varepsilon}}, \overline{r_{\varepsilon}}\right)$. Besides, since $\operatorname{det}\left(\eta\left(x_{3}\right), \dot{\eta}\left(x_{3}\right)\right)$ does not admit any real roots, its modulus is lower-bounded on the real axis and according to (61), one can make $\operatorname{det}\left(\mathcal{M}\left(x_{3}, t\right)\right)$ positive for $x_{3}$ in the compact set $\left[R-\overline{r_{\varepsilon}}, R-\underline{r_{\varepsilon}}\right] \cup\left[R+\underline{r_{\varepsilon}}, R+\bar{r}_{\varepsilon}\right]$ by choosing $\alpha$ sufficiently large. In that case, its roots are in $C\left(R, \underline{r_{\varepsilon}}, \overline{r_{\varepsilon}}\right)$, but not in $C\left(R, \underline{r_{\varepsilon}}, \overline{r_{\varepsilon}}\right) \cap \mathbb{R}$ : they are necessarily complex.

\section{$.5 \quad$ Proof of Theorem 6}

The result is proved by applying Rouché's theorem on (38) with path $\Gamma_{\underline{r_{\varepsilon}}}(R)$. To do this, we need to lower-bound $\left|Q\left(x_{3}\right)\right|$ and upper-bound the term $O\left(\lambda^{3}\right)$ in (38). When $\left|i_{q}\right| \neq \frac{1-\varepsilon}{2}|i|, Q$ does not have any root on $\Gamma_{\underline{r_{\varepsilon}}}(R)$ and $|Q|$ can thus be lower-bounded in this set by some $\underline{d}_{2}>0$. As for the term $O\left(\lambda^{3}\right)$, by following the proof of Lemma 2 , the reader can check that it is actually a rational function in $x_{3}$ whose coefficients are bounded in time, at least for $t \geq \bar{t}_{1} \frac{\ln \alpha}{\alpha}$ (with $\bar{t}_{1}$ some scalar independent from $\alpha$ ) and whose denominator is necessarily of the form $\operatorname{det}\left(\mathcal{M}\left(x_{3}, t\right)\right)^{k_{1}} \operatorname{det}\left(\eta\left(x_{3}\right), \dot{\eta}\left(x_{3}\right)\right)^{k_{2}}$ (coming from the inversion of the corresponding matrices). On $\Gamma_{\underline{r_{\varepsilon}}}(R), \operatorname{det}\left(\eta\left(x_{3}\right), \dot{\eta}\left(x_{3}\right)\right)$ does not have any root and can be lower-bounded. The same thing holds for $\operatorname{det}\left(\mathcal{M}\left(x_{3}, t\right)\right)$ uniformly in time for $t \geq \bar{t}_{0} \frac{\ln \alpha}{\alpha}$ by taking $\alpha$ sufficiently large according to (61). Therefore, the term $O\left(\lambda^{3}\right)$ in (38) can be upper-bounded by a polynomial of $\left|x_{3}\right|$ for $t \geq \bar{t}(\alpha)=\max \left\{\bar{t}_{0}, \bar{t}_{1}\right\} \frac{\ln \alpha}{\alpha}$. More precisely, denoting $\gamma_{2}=\tilde{\lambda}_{1}^{4}+\tilde{\lambda}_{2}^{4}+\tilde{\lambda}_{3}^{4}$, there exists a polynomial $\mathfrak{R}_{2}$ (time and $\alpha$ independent) such that for all $t \geq \bar{t}$ and all $x_{3}$ in $\Gamma_{\underline{r_{\varepsilon}}}(R)$,

$$
\begin{aligned}
\left|\frac{1}{\alpha^{4}} J\left(x_{3}, t\right)-\gamma_{2} Q\left(x_{3}\right)\right| & \leq \frac{\mathfrak{R}_{2}\left(\left|x_{3}\right|\right)}{\alpha} \\
& \leq \frac{\bar{\Re}_{2}}{\alpha} \leq \gamma_{2} \underline{d}_{2} \leq\left|\gamma_{2} Q\left(x_{3}\right)\right|
\end{aligned}
$$

Since $\mathcal{M}(\cdot, t)^{-1}$ is defined on $B_{\underline{r_{\varepsilon}}}(R)$ (where its determinant does not have any root), $J(\cdot, t)$ is

\footnotetext{
${ }^{4} B_{r}(a)$ denotes the ball with center $a$ and radius $r$.
} 
holomorphic on that set and according to Rouché's theorem, it admits as many zeros as $Q$ on $B_{\underline{r_{\varepsilon}}}(R)$, i.e. either one or two depending on $i_{q}$.

\section{References}

[1] P. Acarnley and J. Watson, "Review of position-sensorless operation of brushless permanentmagnet machines," IEEE Transactions on Industrial Electronics, vol. 53, no. 2, pp. 352-362, 2006.

[2] F. Poulain, L. Praly, and R. Ortega, "An observer for permanent magnet synchronous motors with currents and voltages as only measurements," IEEE Conference on Decision and Control, 2008 .

[3] J. Lee, J. Hong, K. Nam, R. Ortega, L. Praly, and A. Astolfi, "Sensorless control of surfacemount permanent-magnet synchronous motors based on a nonlinear observer," IEEE Transactions on Power Electronics, vol. 25, no. 2, pp. 290-297, 2010.

[4] R. Ortega, L. Praly, A. Astolfi, J. Lee, and K. Nam, "Estimation of rotor position and speed of permanent magnet synchronous motors with guaranteed stability," IEEE Transactions on Control Systems Technology, vol. 19, no. 3, pp. 601-614, 2011.

[5] J. Malaizé, L. Praly, and N. Henwood, "Globally convergent nonlinear observer for the sensorless control of surface-mount permanent magnet synchronous machines," IEEE Conference on Decision and Control, 2012.

[6] N. Henwood, J. Malaizé, and L. Praly, "A robust nonlinear Luenberger observer for the sensorless control of SM-PMSM : Rotor position and magnets flux estimation," IECON Conference on IEEE Industrial Electronics Society, 2012.

[7] A. Bobtsov, A. Pyrkin, and R. Ortega, "A new approach for estimation of electrical parameters and flux observation of permanent magnet synchronous motors," Int. J. Adapt. Control Signal Process., vol. 30, pp. 1434-1448, 2015.

[8] P. Bernard and L. Praly, "Robustness of rotor position observer for permanent magnet synchronous motors with unknown magnet flux," IFAC World Congress, 2017.

[9] Y.-S. Han, J.-S. Choi, and Y.-S. Kim, "Sensorless pmsm drive with a sliding mode control based aadaptive speed and stator resistance estimator," IEEE Transactions on Magnetics, vol. 36, no. 5, pp. 3588-3591, 2000.

[10] B. Nahid-Mobarakeh, F. Meibody-Tabar, and F.-M. Sargos, "Mechanical sensorless control of pmsm with online estimation of stator resistance," IEEE Transactions on Industry Applications, vol. 40, no. 2, pp. 457-471, 2004. 
[11] M. Hinkkanen, T. Tuovinen, L. Harnefors, and J. Luomi, "A combined resistance and statorresitance observer for salient pmsm drive: design and staility analysis," IEEE Transactions on Power Electronics, vol. 27, no. 2, pp. 601-609, 2012.

[12] C. Verrelli, P. Tomei, E. Lorenzani, G. Migliazza, and F. Immovilli, "Nonlinear tracking control for sensorless permanent magnet synchronous motors with uncertainties," Control Engineering Practice, vol. 60, pp. 157-170, 2017.

[13] — - "Persistency of excitation and position-sensorless control of permanent magnet synchronous motors," Automatica, vol. 95, pp. 328-335, 2018.

[14] J.-G. Romero, R. Ortega., Z. Han, T. Devos, and F. Malrait, "An adaptive flux observer for the permanent magnet synchronous motor," Int. J. Adapt. Control Signal Process., vol. 30, pp. 473-487, 2016.

[15] P. Vaclavek, P. Blaha, and I. Herman, "AC Drive Observability Analysis," IEEE Transactions on Industrial Electronics, vol. 60, no. 8, pp. 3047-3059, 2013.

[16] J. A. Moreno, H. Mujica-Ortega, and G. Espinosa-Pérez, "A global bivalued-observer for the sensorless induction motor," IFAC World Congress, 2017.

[17] C. Verrelli, E. Lorenzani, R. Fornari, M. Mengoni, and L. Zarri, "Steady-state speed sensor fault detection in induction motors with uncertain parameters: A matter of algebraic equations," Control Engineering Practice, vol. 80, pp. 125-137, 2018.

[18] C. M. Verrelli and P. Tomei, "AC motors: letter swap potentialities," Automatica, vol. 113, p. $108763,2020$.

[19] J. Moreno and G. Besançon, "Multivalued finite-time observers for a class of nonlinear systems," IEEE Conference on Decision and Control, 2017.

[20] D. Luenberger, "Observing the state of a linear system," IEEE Transactions on Military Electronics, vol. 8, pp. 74-80, 1964.

[21] N. Kazantzis and C. Kravaris, "Nonlinear observer design using Lyapunov's auxiliary theorem," Systems and Control Letters, vol. 34, pp. 241-247, 1998.

[22] V. Andrieu and L. Praly, "On the existence of a Kazantzis-Kravaris / Luenberger observer," SIAM Journal on Control and Optimization, vol. 45, no. 2, pp. 432-456, 2006.

[23] P. Bernard, "Luenberger observers for nonlinear controlled systems," IEEE Conference on Decision and Control, 2017.

[24] P. Bernard and V. Andrieu, "Luenberger observers for non autonomous nonlinear systems," Transactions on Automatic Control, 2018. 
[25] J. Chiasson, Modeling and High-Performance Control of Electric Machines. John Wiley \& Son, 2006.

[26] P. Bernard and L. Praly, "Estimation of position and resistance of a sensorless PMSM : a nonlinear luenberger approach for a non-observable system," Tech. Rep., 2020. [Online]. Available: https://hal.archives-ouvertes.fr/hal-01824171/

[27] N. Henwood, "Estimation en ligne de paramtres de machines lectriques pour vhicule en vue d'un suivi de la temprature de ses composants," Ph.D. dissertation, Control and System Center, MINES ParisTech, https://pastel.archives-ouvertes.fr/pastel-00958055, 2014.

[28] M. Bodson and J. Chiasson, "Differential-geometric methods for control of electric motors," Int. J. Robust. Nonlinear Control, vol. 8, pp. 923-254, 1998. 